Wright State University

CORE Scholar

2012

\title{
Enhanced Angiotensin II-Induced Cardiac and Aortic Remodeling in ACE2 Knockout Mice
}

Mahmoud Alghamri

Wright State University

Follow this and additional works at: https://corescholar.libraries.wright.edu/etd_all

Part of the Pharmacology, Toxicology and Environmental Health Commons

\section{Repository Citation}

Alghamri, Mahmoud, "Enhanced Angiotensin II-Induced Cardiac and Aortic Remodeling in ACE2 Knockout Mice" (2012). Browse all Theses and Dissertations. 1090.

https://corescholar.libraries.wright.edu/etd_all/1090

This Thesis is brought to you for free and open access by the Theses and Dissertations at CORE Scholar. It has been accepted for inclusion in Browse all Theses and Dissertations by an authorized administrator of CORE Scholar. For more information, please contact library-corescholar@wright.edu. 


\title{
Enhanced Angiotensin II-Induced Cardiac and Aortic Remodeling in ACE2 Knockout Mice
}

\author{
A thesis submitted in partial fulfillment \\ of the requirements for the degree of \\ Master of Science \\ By \\ MAHMOUD ALGHAMRI \\ B. Pharm, Alazhar University, Gaza, Palestine 2008
}

2012

Wright State University 



\title{
WRIGHT STATE UNIVERSITY \\ SCHOOL OF GRADUATE STUDIES
}

Date: August 1, 2012

I HEREBY RECOMMEND THAT THE THESIS PREPARED UNDER MY

SUPERVISION BY Mahmoud Alghamri ENTITLED "Enhanced Angiotensin II-Induced

Cardiac and Aortic Remodeling in ACE2 Knockout Mice” BE ACCEPTED IN

PARTIAL FULFILLMENT OF THE REQUIREMENTS FOR THE DEGREE OF

Master of Science.

\author{
Mariana Morris, Ph.D. \\ Thesis Director, Chair \\ Department of Pharmacology and Toxicology
}

Terry Oroszi

Director, Graduate Program

Committee on Final Examination

Mariana Morris, Ph.D.

Mark Anstadt, M.D, FACS.

Roberta L. Pohlman, Ph.D.

Andrew T. Hsu, Ph.D.

Dean, School of Graduate Studies 



\begin{abstract}
Mahmoud Alghamri. M.S., Department of Pharmacology and Toxicology, Wright State University, 2012. Enhanced Angiotensin II-Induced Cardiac and Aortic Remodeling in ACE2 Knockout Mice.
\end{abstract}

Activation of the renin angiotensin system (RAS) is a well-known driving force, governing the aggravation and progression of CVD and associated target organ damage. Angiotensin (Ang) converting enzyme 2 (ACE2), a new member within the RAS family, was first cloned from the ventricle of patient with heart failure. ACE2 was shown to be upregulated under pathological conditions. We hypothesize that loss of ACE2 negatively affects cardiac function and exacerbates hypertrophic cardiomyopathy and aortic remodeling in response to Ang II. Eight weeks old ACE2 knock out (KO) and wild type (WT) mice were infused with Ang II s.c. (1000 ng/kg/min for 4 weeks) using osmotic pumps. Blood pressure (radiotelemetry), cardiac function (echocardiography) and cardiac/aortic structural damage (histology) were examined. At baseline before Ang II, ACE2 KO displayed a normal phenotype for cardiac function and blood pressure. After 4 weeks of Ang II infusion, the mean arterial pressure (MAP) was increased ( $40 \%$ in WT and $\sim 33 \%$ in ACE2 KO) with no differences between groups. However, ACE2 KO responded differently to the increased MAP. Cardiac function analysis revealed severe myocardial dysfunction shown by the lowered EF\% (31\% vs. 13\%) as well as FS\% (30\% vs 6\%) in ACE2 KO vs WT, respectively. In addition, the cardiac dysfunction was associated with hypertrophic cardiomyopathy shown by the increased left ventricular wall thickness as well as heart weight/ body weight ratio. Moreover, collagen staining in the myocardium and aorta revealed higher collagen percentage in ACE2 KO which indicates 
cardiovascular remodeling. Furthermore, results showed enhanced oxidative stress in the myocardium and aorta of Ang II infused ACE2 KO compared to Ang II WT. In conclusion, ACE2 attenuates myocardial maladaptive hypertrophy and cardiovascular remodeling in response to long term Ang II stimulation. 
1. INTRODUCTION .......................................................... 1

Renin angiotensin system (RAS) ................................... 1

Angiotensin II (Ang II) ............................................... 2

Angiotensin converting enzyme 2 (ACE2) ........................... 5

Echocardiography.................................................... 8

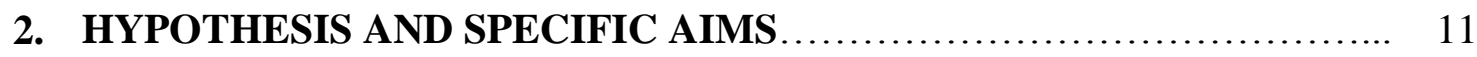

3. MATERIALS AND METHODS ..................................... 12

Experimental protocol.............................................. 12

Animals................................................................ 12

Surgical insertion of radiotelemetric probes ................................ 14

Angiotensin II infusion................................................. 14

Measurement of blood pressure........................................ 14

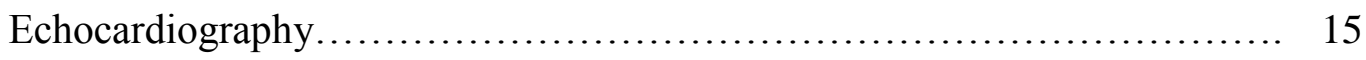

Haematoxylin \& Eosin staining and cardiomyocyte size quantification...... 16

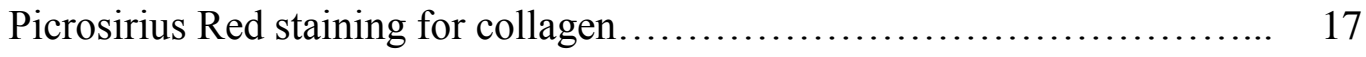

Picrosirius Red quantitation......................................... 17

Quantitation of the interior lumen of the aorta........................ 18

Dihydroethidium fluorescence for reactive oxygen species................ 18

Dihydroethidium staining quantification............................... 19

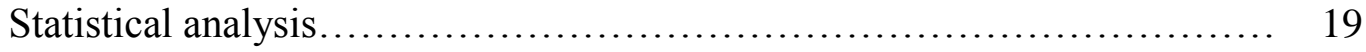

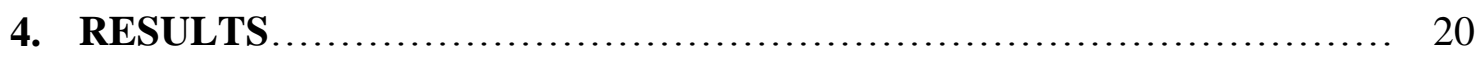

Baseline cardiac function and blood pressure in ACE2 KO.............. 20

Cardiac function and blood pressure post Ang II infusion ................. 20

Cardiomyocyte size quantitation........................................................ 21 
Evidence for cardiac and aortic fibrosis in Ang II infused ACE2 KO...... 21

Masson's Trichrome staining for collagen.......................... 21

Picrosirius Red staining for collagen fibers......................... 21

DHE staining for superoxide.................................... 22

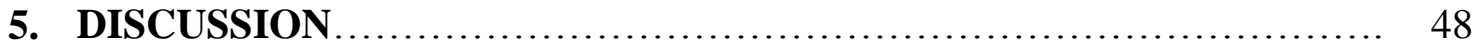

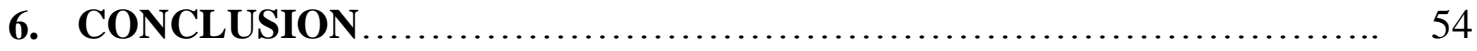

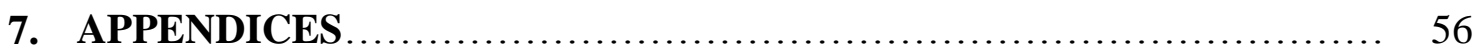

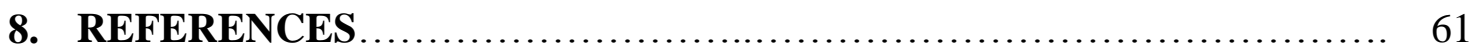


1. Angiotensin II effects on different body organs........................... 4

2. Different enzymatic pathways interfere with RAS....................... 7

3. Generation of ACE2 KO mice..................................... 13

4. Baseline Echocardiography......................................... 26

5. Echocardiographic assessments after 4 weeks of Ang II................... 27

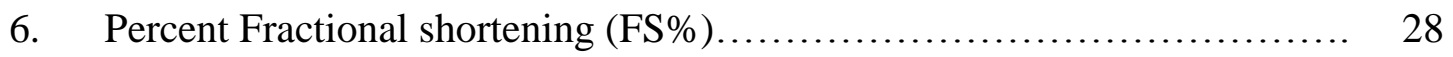

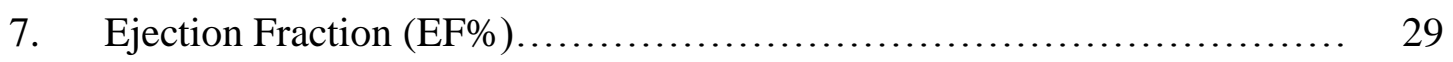

8. E/A Ratio ...................................................... 30

9. Mean Arterial Pressure (MAP) ......................................... 31

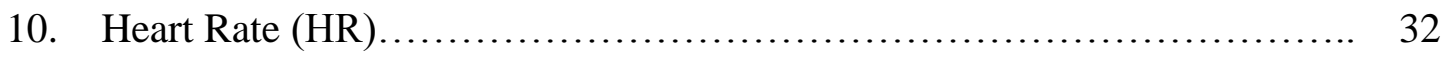

11. Cardiomyocyte area Quantitation......................................... 33

12. Heart weight/ Body Weight........................................ 35

13. Masson's Trichrome staining of collagen (Heart)........................ 37

14. Picrosirius Red Staining (Heart) .................................... 38

15. Picrosirius Red Staining (Heart) ....................................... 39

16. Collagen Volume Fraction (CVF \%)................................... 40

17. Picrosirius Red Staining (Aorta) ....................................... 41

18. Collagen\% (Aorta) ................................................ 42

19. Luminal area of the aorta ............................................... 43

20. Myocardial DHE fluorescence....................................... 44

21. Reactive Oxygen Species \% (heart) ................................. 45

22. DHE fluorescence (aorta) .......................................... 46

23. Reactive Oxygen Species \% (aorta) ................................. 47 
1. Detailed Echo data analysis of cardiac function in the WT and ACE2 KO at the baseline and after 4 weeks of Ang II stimulation.................... 24 


\section{INTRODUCTION}

\section{Renin Angiotensin System (RAS):}

There is speculation that cardiovascular disease (CVD) will be the leading cause of death by 2020 (Burrell, Johnston, Tikellis, \& Cooper, 2004). Activation of the renin angiotensin system (RAS) is well-known as the driving force that governs aggravation and progression of CDV and the associated target organs damage. The release of Angiotensinogen hormone from the liver is the precursor from which the subsequent hormone peptides, namely Ang I and Ang II by the action of renin and Angiotensin (Ang) Converting Enzyme (ACE), respectively, will be formed. Inhibition of Ang II formation or subsequent binding to the AT1 receptor is a key target in current pharmacotherapeutics for CVD.

Renin was first reported to be the causative agent of hypertension in hypertensive rats in 1898 (Kunikullaya, Ananthakrishnan, \& Goturu, 2011). Later on, in1934, Goldblatt et al., found that hypertension in animal models induced by renal ischemia caused a release of vasoconstrictor substance (Goldblatt, Lynch, Hanzal, \& Summerville, 1934). After that, two independent groups were the first to report the end product of this cascade in 1939 and they named it as "Hypertensin" (Fasciolo, Houssay, \& Taquini, 1938) and "Angiotonin" (Page, 1939), separately. Soon after, the two groups agreed on a common nomenclature of the vasopressor end product of renin activity as (angiotensin) (BraunMenendez \& Page, 1958). It was not till 1956 when today’s RAS was described (Skeggs., Kahn, \& Shumay, 1956). Angiotensin Converting Enzyme (ACE) was first discovered in the plasma (Skeggs, Jr. et al., 1956). As a result, the conversion of Ang I to Ang II was thought to take place predominantly in the plasma. However, it was found that most of 
Ang II formation occurs in the pulmonary circulation rather than in the plasma $(\mathrm{Ng} \&$ Vane, 1967). The main antihypertensive effect of ACE inhibitor is mediated by decreasing plasma Ang II level (Melaragno \& Fink, 1996).

\section{Angiotensin II}

As a main member in the renin angiotensin system (RAS), Angiotensin (Ang) II, also known as Ang (1-8) $1046 \mathrm{~m} / \mathrm{z}$, is one of the essential hormone peptides that regulate hemodynamic body functions. It has a wide variety of effects on various body organs. In addition, its overproduction is responsible for the progression and complication of most of cardiovascular disorders. Research studies have been conducted over decades trying to explore the effects of this harmful substance on body organ function.

The exact role of Ang II in the complication of CVD has been studied for decades. Longterm Ang II infusion is often used as an experimental model for essential hypertension, resulting in a gradual and sustained increase in blood pressure and multiple organ pathologies (Kirchhoff et al., 2008; Xu, Zhi, Hou, Cohen, \& Jiang, 2011; Ruiz-Ortega et al., 2001a). The way in which Ang II induces hypertension has been explained by many mechanisms, including increasing peripheral vascular resistance, stimulating electrolyte and fluid retention either directly through Ang II type I (AT $\left.)_{1}\right)$ receptor (de Gasparo, 2002), or indirectly by stimulating aldosterone and vasopressin secretion. Excessive Ang II has deleterious effects on myocardium by a plethora of mechanisms such as increasing sarcolemmic permeability and death of cardiac myocytes or increasing permeability and destruction of coronary microvascular endothelial cells (Gavras \& Gavras, 2002a). Other mechanisms include mediating oxidative stress via activation of NADPH through $\mathrm{AT}_{1}$ 
receptor activation and the subsequent formation of ROS (Kassiri et al., 2009a) or via endothelin-1 peptide activation (Xia \& Karmazyn, 2004). It has been documented that cardiac myocytes undergo hypertrophy as well as fibrosis associated with collagen production in response to Ang II both in vivo (Grobe et al., 2007) and in vitro (Wang et al., 2010).

Ang II infusion is associated with increased pro-inflammatory mediators in the vascular smooth muscle cells (Ruiz-Ortega et al., 2001b). In addition, $\mathrm{AT}_{1}$ receptor activation mediates a growth response in several cell types, in particular, endothelial cells and cardiomyocytes (de Gasparo, Catt, Inagami, Wright, \& Unger, 2000). Moreover, AT $_{1}$ receptor blockers are effective in decreasing interstitial cardiac fibrosis in the failing heart (De Mello \& Specht, 2006). A study of the effects of Ang II in adult rats showed myocyte hypertrophy and midmyocardial interstitial fibrosis (Grobe et al., 2007). Ang II mediated cardiac remodeling is facilitated through myocardial $\mathrm{AT}_{1}$ receptors and subsequent intracellular signaling (Browe \& Baumgarten, 2004; de Gasparo et al., 2000). Interestingly, cardiac Ang II overexpression is associated with left ventricular hypertrophy, yet is independent of the effects of pressure overload (Mazzolai et al., 1998). Collectively, results point toward a detrimental role of elevated Ang II in the myocardium. 


\section{Effects of Angiotensin II}

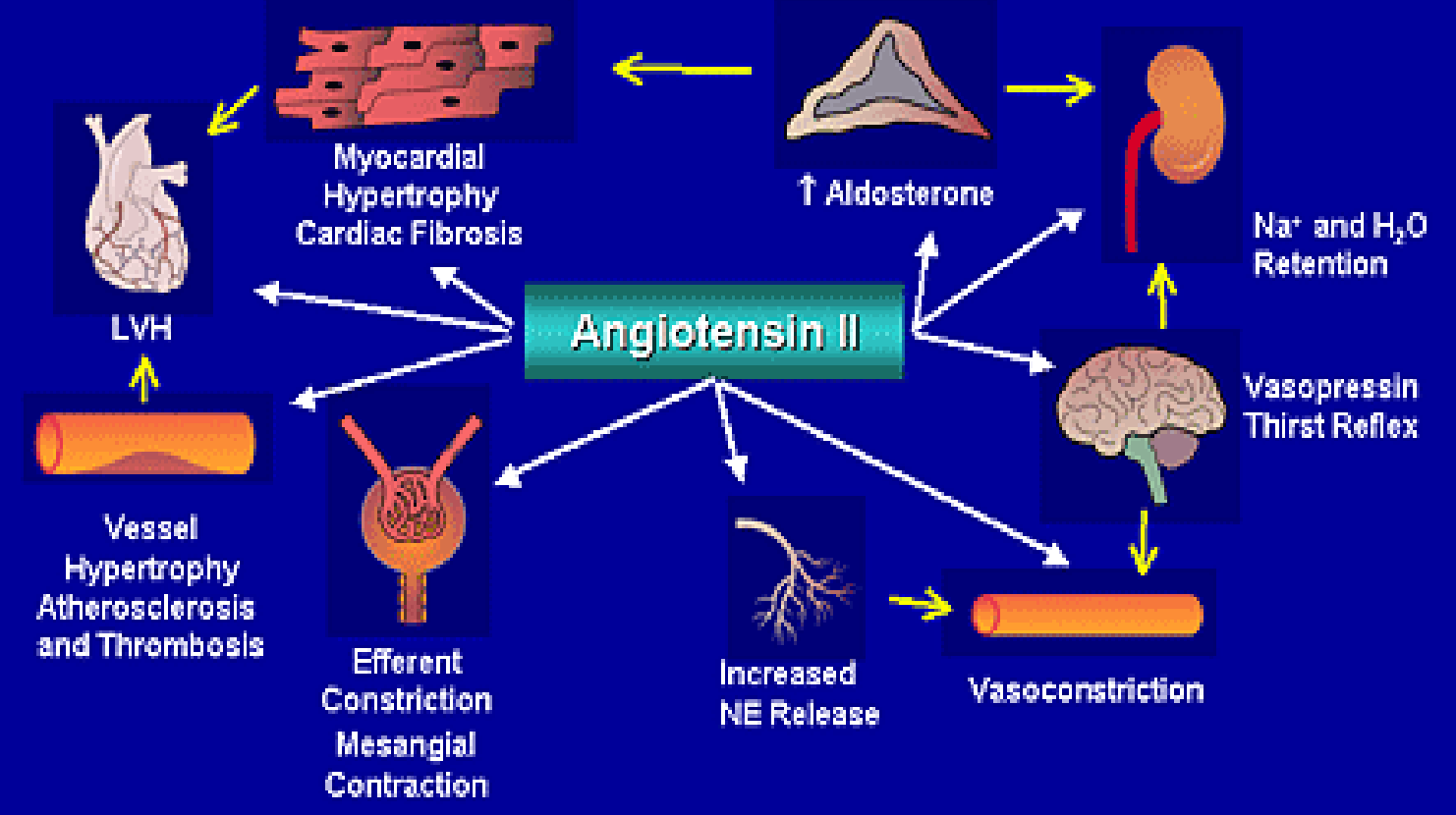

$\mathrm{LVH}=$ left ventricular hypertrophy; NE = norepinephnine.

Figure 1. Angiotensin II effects on different body organs. (Modified from http://www.medscape.org) 


\section{Angiotensin Converting Enzyme 2 (ACE2):}

The discovery of a new member within the RAS family, ACE2 , named due to $40 \%$ homology and $61 \%$ sequence similarity with ACE, by two different groups in 2000 (Donoghue et al., 2000; Tipnis et al., 2000) caused much excitement in the field of CVD pharmacotherapy. The main action of ACE2 is to shuttle Ang II and to tip the balance toward Ang (1-7) formation (Xu, Sriramula, \& Lazartigues, 2011). Functionally, ACE2 is a pleiotropic monocarboxypeptidase and metabolizes a range of peptides (Zhong et al., 2010). Although its primary action is to convert Ang I to Ang (1-9) (Donoghue et al., 2000), ACE2 catalytic efficiency is 400-fold higher with Ang II as a substrate than with Ang I (Vickers et al., 2002). In addition, the metabolic product of this enzymatic activity, Ang (1-7), is a well-known peptide to counteract the deleterious Ang II mediated effects (Ferrario, Chappell, Tallant, Brosnihan, \& Diz, 1997). Thus, ACE2 is believed to have a pivotal role in cardiovascular disease progression as a negative regulator of the RAS family. The fact that ACE2 was first found in heart failure ventricles (Donoghue et al., 2000) and with its predominant expression in the heart (Tipnis et al., 2000), together, fueled researchers with tremendous incentives to explore the essential role of this enzyme in heart function.

It has been reported that ACE2 null mice display impaired cardiac contractility function in comparison to their control littermates. This result was associated with a severe decrease in the percent fractional shortening with higher LVESD and LVEDD in those mice, an effect mediated by Ang II, (Crackower et al., 2002; Oudit et al., 2007; Nakamura et al., 2008). Two different studies of the ACE2 level in failing hearts showed that ACE2 protein level was elevated in the infarcted myocardial tissue (Zisman et al., 
2003; Kassiri et al., 2009b). Moreover, this increase limits the adverse effects of the raised Ang II by an increasing level of vasodilator Ang (1-7) (Burrell et al., 2004). In addition, a study of the ACE2 gene in the myocardium of patients with end stage heart failure showed that the amount of ACE2 mRNA positively correlated with left ventricular end-systolic diameter (Ohtsuki et al., 2010). Despite the variations seen in the phenotype of ACE2 null mice with mixed genetic background in the later studies, none of these studies negate either the fact that ACE2 is the key pathway for the metabolism of Ang II in vivo (Gurley \& Coffman, 2008) or that loss of ACE2 aggravates heart failure (Ferreira et al., 2010). Collectively, these findings demonstrate that loss of ACE2 is accompanied by impaired cardiac function, especially in the case of pathological conditions. 


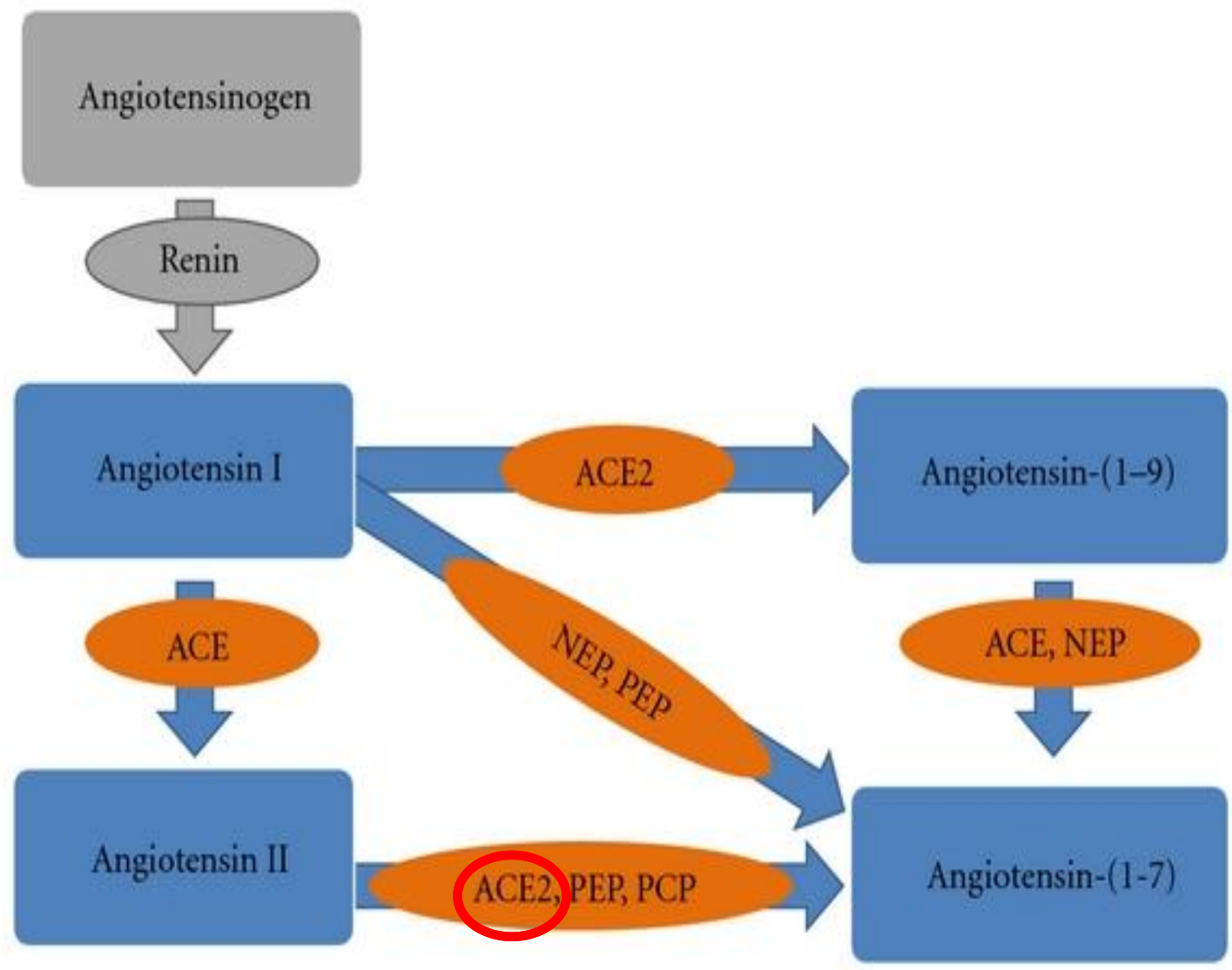

Figure 2. Different enzymatic pathways interfere with RAS. (Modified from Gomes et al., 2012) 


\section{Echocardiography:}

Traditionally, a diversity of techniques have been elaborated over decades to assess cardiac functions including, microangiography, ventricular pressure-volume catheterization, and thermo-dilution techniques in mice. However, those techniques are invasive since they require instrumentation as well as surgical intervention which limit the reliability of serial measurement due to the physiological hemodynamic changes (Collins, Korcarz, \& Lang, 2003; Pacher, Nagayama, Mukhopadhyay, Batkai, \& Kass, 2008). The quest for a noninvasive, reliable and reproducible method to assess cardiac structure and function in rodents was a challenge for researchers, especially, owing to the small heart size and rapid heart rate (around 600 bpm) for those murines (Liu \& Rigel, 2009). The adaptation of echo assessment to murine models is considered an expedient technique for the in vivo assessment of cardiac function. Adding to that, the progressive enhancement in image acquisition using the merits of the newly developed linear transducers with high frequency and the digitalized software for image processing, dramatically increased the scope of echo assessment in murines. One of the earliest applications of clinical echocardiography was in the evaluation of left ventricular (LV) size and function (Picard, Popp, \& Weyman, 2008). Moreover, the evolution of the left ventricular long-axis measurement and the area measurements of the ventricular contours incrementally increased echocardiographic accuracy (Picard et al., 2008).

Echocardiography (echo) (sonogram of the heart) is a non-invasive, highly reliable, and accurate technique for assessment of cardiac structure and function in mice (Tanaka et al., 1996; Gao, Dart, Dewar, Jennings, \& Du, 2000). Hartley et al. (2002) suggested that cardiac ultrasound is the most accurate technique to obtain cardiovascular imaging that is 
suitable for murine application (Hartley, Taffet, Reddy, Entman, \& Michael, 2002). Moreover, in 1995, Gardin et.al was able to assess systolic dysfunction accurately in mice (Gardin, Siri, Kitsis, Edwards, \& Leinwand, 1995). One of the most commonly used methods for echocardiographic procedures is transthoracic echocardiography (TTE), which allows the heart to be viewed in 2-Dimension (2-D) by applying a transducer directly on the chest. The advantage of TTE over other echocardiographic techniques is that it is non-invasive and includes the feasibility to do serial assessment. Each view has its own mode from which accurate measurements of the cardiac parameters can be obtained. During the parasternal long-axis view, for instance, 2-D mode represents the most accurate measurement of the LV mass in mice (Youn et al., 1999). On the other hand, short axis 2-D mode and 2-D guided M-mode are recognized as reliable methods in identification of impaired systolic function (Tanaka et al., 1996). Pulse Wave Doppler (PWD) has been evaluated in many studies to be a reliable method in evaluation of mitral valve function, a strong indicator of LV diastolic function (Sohn et al., 1999; Tanaka, 2003; Vlahovic \& Popovic, 1999). Furthermore, along with color flow imaging, PWD is considered a useful tool in the assessment of cardiac remodeling (Patten, Aronovitz, Bridgman, \& Pandian, 2002).

Among the parameters for the assessment of cardiac function, fractional shortening (FS\%) and Ejection Fraction (EF\%) are the most commonly used indexes of global systolic function (Liu \& Rigel, 2009). Schiller et al. (1996) reported that EF\% is the most commonly discussed index for LV function Moreover, Kuecherer et al. (1991) posited that $\mathrm{EF} \%$ has the least variability, and thus, the most reproducible index for the evaluation of LV contractility function. FS\% has been used for LV systolic function 
assessment (Gardin et al., 1995). A study done by Gao et al. (2000) on mice heart post MI showed that FS\% was significantly correlated with LV systolic function and the decrease in FS\% was parallel with LV enlargement in those mice (Gao et al., 2000). Emilsson et al. (2006) suggested there was a good correlation between EF\% and FS\% and thus, confirmed to be a suitable index for the assessment of LV systolic function (Emilsson, Egerlid, Nygren, \& Wandt, 2006). 


\section{HYPOTHESIS AND SPECIFIC AIMS}

\subsection{Hypothesis}

For this study, it is hypothesized that the loss of ACE2 negatively affects cardiac function and exacerbates hypertrophic cardiomyopathy and aortic remodeling in response to Ang II.

\subsection{Specific aims}

Two specific aims are associated with this study.

1. To test the hypothesis that deletion of ACE2 accentuates Ang II mediated cardiac dysfunction.

2. To test the hypothesis that loss of ACE2 enhances Ang II mediated cardiac and vascular structural maladaptation. 


\section{Materials and Methods}

\subsection{Experimental protocol:}

Mice ( 8 weeks old) were anesthetized and telemetric probes were inserted in the carotid artery for blood pressure (BP) recording. After a minimum of one week recovery, mice were infused with Ang II (1000 ng/kg/min) for 4 weeks using Alzet ${ }^{\circledR}$ osmotic pumps (ALZA, CO., Cupertino, CA). Cardiac function and mean arterial pressure (MAP) were assessed at baseline and after 4 weeks of Ang II infusion by Echo and telemetry, respectively. At the end of the peptide infusion, mice were either decapitated or perfused with $4 \%$ paraformaldehyde. Hearts and aortas were quickly removed and frozen in liquid nitrogen or fixed in $4 \%$ paraformaldehyde. All experimental protocols were approved by the Wright State University (WSU) Animal Care and Use Committee. Fixed hearts and aortas were sent to a commercial laboratory (AML Laboratories, Inc., Baltimore, MD) for paraffin embedding, sectioning, and staining.

\subsection{Animals:}

C57B1/6 male mice ( 8 weeks old, WT and ACE2 KO, n=7-8 per group) were housed at $22^{\circ} \mathrm{C}$ under a 12 -hour light/12-hour dark cycle with ad libitum access to tap water and standard mouse chow (29\% protein, 17\% fat, 54\% carbohydrate, diet 8640$)$ (Teklad Animal Diets, Harlan Labs, USA). ACE2 KO male and ACE2 heterozygous female breeding pairs were a gift from Drs. Coffman and Gurley (Duke University). Mice were genotyped for ACE2 gene expression. Generation of the C57B1/6 ACE2 KO strain has been described previously (Gurley et al., 2006). 


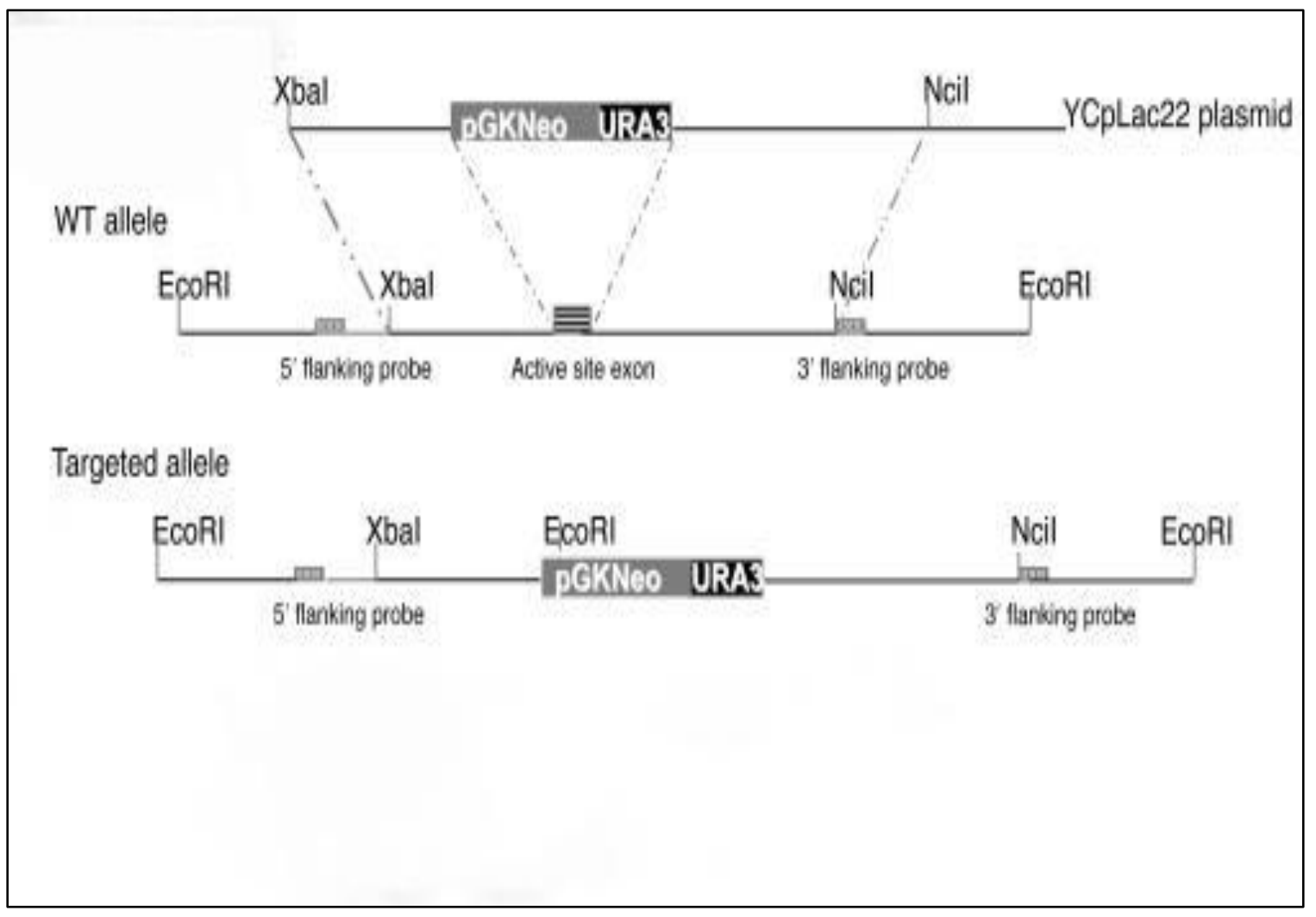

Figure 3. Generation of ACE2 KO mice. (Modified from Gurley et al., 2006) 


\subsection{Surgical insertion of radiotelemetric probes:}

Mice were anesthetized with ketamine/xylazine IM $(75 \mathrm{mg} / \mathrm{kg}$ ketamine and $7.5 \mathrm{mg} / \mathrm{kg}$ xylazine) (Butler Co., Columbus, OH). A radiotelemetric probe, model TA11PA-C20 (Data Sciences International, St. Paul, MN) was inserted into left carotid artery and advanced into the aortic arch. Carprofene (5mg/kg) (Sigma-Aldrich, St. Louis, MO) was administered s.c. for pain management immediately after the surgery and at 24 hrs postsurgery. Heating pads were used to maintain body temperature and saline drops were administered to the eyes periodically to prevent dryness of the sclera. Animals were allowed at least one week of recovery before any experiments were conducted.

\subsection{Ang II infusion:}

Ang II (Bachem Inc., Torrance, CA) was dissolved in sterile saline and loaded into osmotic pumps (model 1004, ALZA, CO., Cupertino, CA). Pumps were kept at $37{ }^{\circ} \mathrm{C}$ in sterile saline solution for overnight. The Ang II dose was $1000 \mathrm{ng} / \mathrm{kg} / \mathrm{min}$ for 4 weeks. For pump insertion, mice were anesthetized with isoflurane (3\% induction box, $1.5 \%$ maintenance using a nose cone). Heating pads were used to maintain body temperature. The upper back was shaved and cleaned 3x with betadine/70\% ethanol. A small transverse incision was made between the scapulae and the pump was inserted (cap first) in a s.c. pocket on the left lower flank. Carprofen $(5 \mathrm{mg} / \mathrm{kg})$ was administered s.c. for analgesia as described. 


\subsection{Measurement of blood pressure:}

Radiotelemetry was used to measure BP continuously $(500 \mathrm{~Hz})$ for $24 \mathrm{hr}$ periods at baseline and during Ang II infusion on a weekly basis. The analysis system used was the standard Dataquest A.R.T. system (Data Sciences International, St. Paul, MN).

\subsection{Echocardiography:}

Echocardiographic (Echo) measurements were recorded using a Siemens® ACUSON Sequoia 512 ultrasound system (Siemens Medical Solutions USA, Inc., Malvern, PA) with an ACUSON 15 megahertz transducer (model 15L8, Siemens Medical Solutions Inc., Malvern, PA). Mice were anesthetized with $3 \%$ isoflurane in $1 \mathrm{~L} /$ min pure oxygen (Surgivet Anesco ${ }^{\circledR}$ Isotec $4^{\mathrm{TM}}$ ). Anesthesia was maintained with $1.5 \%$ isoflurane using a nose cone. Mice were placed in a supine position and body temperature was maintained using a heating pad. Chest hair was removed with an electric shaver and a commercial hair removal cream (Nair®, Church \& Dwight Co., Inc, Township, NJ). The plate was tilted 60 degree anterio-rostrally from the vertical axis. Pre-warmed ultrasound transmission gel (Aquasonic ${ }^{\mathrm{TM}}$ 100, Parker Laboratories Inc., Fairfield, NJ) was applied to the chest. The transducer was positioned at a 90 degree angle to the chest since this position provided optimal recordings.

Initially, 2-Dimentional (2-D) video clips were recorded during the parasternal long axis view. The short axis view was then obtained and 2-D video clips as well as 2-D guided M-mode (2-D M-mode) images of the interior LV wall were recorded. Images were standardized by the aortic annulus and the papillary muscles during the long axis and short axis views, respectively. The EF\% was calculated based on the end systolic and 
diastolic volume measurement by the integrative function using the modified Simpson's formula of areas along with major axes (calculated by the Echo). During the transthoracic short axis 2-D M-mode, the left ventricular end systolic diameter (LVESd) and end diastolic diameters (LVEDd) as well as septal wall thickness (SWT) and posterior wall thickness (PWT) were measured. FS\% was calculated using the formula $F S \%=$ $[(L V E D d+L V E S d) / L V E D d] * 100$. Whereas the relative wall thickness (RWT) was calculated using the formula $R W T=[(S W T+P W T) / L V E D d]$. Mitral valve function was assessed during PWD mode. Images were obtained by tilting the mice backwards (posterio-caudally) and directing the probe upward so that the probe was orthogonal with the apex of the heart (Respress \& Wehrens, 2010). Isovolumetric contraction time (IVCT) and isovolumetric relaxation time (IVRT) were also measured. Finally, E-wave and A-wave velocities were measured and E/A ratio was calculated.

\subsection{Haematoxylin \& Eosin $(H \& E)$ staining and cardiomyocyte size quantification:}

Slides containing $5 \mu \mathrm{m}$ paraffin embedded sections were de-paraffinized in xylene and hydrated by dipping in ethanol $100 \%, 95 \%, 80 \%, 70 \%, 50 \%$, and water. Then, sections were stained with H\&E for 15 min, followed by washing in running tap water. Slides were allowed to dry on the slide warmer. Finally, sections were cleaned with xylene and covered with a Permount ${ }^{\circledR}$ (Fisher Scientific, Pittsburgh, PA). Pictures of the heart sections were taken at 20X magnification using a Leica Microsystem ${ }^{\circledR}$ DMR microscope. Three separate and random location images of cardiomyocytes were taken in the LV wall (images were taken in gray scale). Quantitation was done by Metamorph ${ }^{\circledR}$ image analysis software (Ver. 7.6.3) using the Threshold selection tool in combination with the smart Integrated Morphometry Analysis (IMA) toolkit. Briefly, thresholds were 
established for the nuclei and for the cardiomyocyte areas. The Area filter was adjusted so that only objects between 30 to 300 pixels were selected (size selected by measuring several nuclei in the control mice), and the Shape filter was set to capture objects with circularity between $0.5-1$ unit. Data were averaged and cell size was computed as total tissue area (pixels) per nucleus.

\subsection{Picrosirius Red staining for collagen:}

Paraformaldehyde fixed, paraffin embedded heart and aorta sections $(5 \mu \mathrm{m})$ were stained with Picrosirius Red for visualization of collagen Type I and III. This staining procedure was performed in-house (WSU Lab). The method used a Weigert's iron haematoxylin staining kit (ENG Scientific, Inc., Clifton, N.J.). After staining for 8 min, slides were washed with tap water and stained with a Picrosirius Red solution containing $5 \mathrm{~g}$ of Direct Red 80 (Sigma-Aldrich, St. Louis, MO) and $500 \mathrm{ml}$ of saturated picric acid solution (Sigma-Aldrich, St. Louis, MO).

\subsection{Picrosirius Red quantification:}

Light microscopic photos were taken at $(2.5 \mathrm{X})$ using polarizing light. The advantage of polarizing light is the high sensitivity which allows for specific detection of the thin collagen fibers. Moreover, this method excludes other possible birefringent fibers. The light source transmitter was connected to the microscope's external port. Polarization was achieved using a specific polarizer and analyzer which were set at 90 degrees to each other in order to restrict light refraction. A section of a control heart was used to calibrate light intensity, plane of polarization, and microscope/camera settings. Once the settings were adjusted, they remained constant for all subsequent imaging. Approximately three 
images were collected for each heart section. Images were taken to include the whole heart section in one image. Quantification was done using Metamorph®7.6.3 with the threshold selection tool as described previously. The threshold was established by adjusting the saturation and intensity of the image to the appropriate level so that it selectively traced the collagen fibers. Image sizes were normalized to 700 pixel width and the threshold was applied to measure the collagen. Total heart area was measured using the trace region selection tool. The collagen volume fraction (CVF \%) was computed as a percentage to the total section area. Quantification of the aorta was completed using the same methods applied to heart.

\subsection{Quantitation of the interior lumen of the aorta:}

Images of sections of the Picrosirius Red stained aorta from both strains were taken at 2.5X. Images were normalized to the same pixels. Quantitation was done by Metamorph ${ }^{\circledR}$ 7.6.3 image analysis software. The luminal areas were traced using the trace region selection tool and the selected areas were measured (Pixels).

\subsection{Dihydroethidium (DHE) fluorescence for reactive oxygen species (ROS):}

DHE (Sigma-Aldrich, St Louis, MO) was used to evaluate the in situ superoxide $\left(\mathrm{O}_{2}^{-}\right)$ level within the myocardium and aorta. DHE is freely permeable to cells and it is oxidized to fluorescent ethidium bromide (EB) in the presence of $\mathrm{O}_{2}^{-}$. EB is a potent mutagen and it is trapped intracellularly by intercalation into the DNA. Hearts were quickly removed and washed in cold PBS. A $3 \mathrm{~mm}$ piece of tissue was trimmed from the left ventricle (using a heart block) and frozen in liquid nitrogen. Frozen myocardium tissue was sectioned $(30 \mu \mathrm{m})$ and thaw mounted onto pre-chilled gel coated slides. One 
mmole/L DHE (Sigma-Aldrich, St Louis, MO) in DMSO (Fisher Scientific, Fair Lawn, $\mathrm{NJ})$ was prepared as a stock solution. It was diluted to $2 \mu$ mole/L in acetone and $10 \mathrm{mM}$ of freshly prepared sodium diethyldithiocarbamate (Sigma-Aldrich, St Louis, MO) was added to inhibit intracellular or extracellular superoxide dismutase (SOD) activity. Sections were incubated with the staining solution at $37^{\circ} \mathrm{C}$ for $30 \mathrm{~min}$. Sections were washed with PBS and cover-slipped using VECTASTIN® hard-set (Vector Laboratories, Inc., Burlingame, CA).

\subsection{DHE staining quantification:}

Quantification of the fluorescence was done using Metamorph ${ }^{\circledR}$ 7.6.3. Areas (400 pixel) within the myocardium and aorta were selected. The threshold was determined by adjusting the intensity level to trace ROS selectively within the myocardium. Regions were normalized by the total selected area.

\subsection{Statistical analysis:}

All data are expressed as mean \pm SEM. Data were analyzed using STATISTICA® 7.1 (StatSoft Inc., Tulsa, OK). Significance was achieved when $p<0.05$. Echo data were analyzed using repeated measure 2 way ANOVA. The rationale for using repeated measure ANOVA is to compare to assess the treatment effect over the time period for both mice strains. The main advantage of this analysis is that it keeps the variability low, since each individual serves as his own control. Modified Tukey post hoc test was used to compare means. Cardiomyocyte size, collagen, and ROS were evaluated using Metamorph® 7.6.3 (Molecular Device, Inc., Sunnyvale, CA) image analysis software as described. Unpaired student's t-test was performed to compare the groups. 


\section{RESULTS}

\subsection{Baseline Cardiac Function and Blood Pressure in ACE2 KO}

Baseline Echo measurements show no differences between WT and ACE2 KO (Figure 4). Baseline 2D M-Mode Echo analysis shows similar FS\% in ACE2 KO as compared to WT (Figure 4; a,b). Results from the long axis 2D view show a slight but non- significant increase in LVESa in ACE2 KO (Figure 4; c,d). However, there was no difference in the calculated EF\%. PWD analysis suggested normal diastolic function with no differences between strains (Figure 4; e,f). In addition, there was no difference in the baseline mean arterial pressure (MAP) between the two strains (Figure 9). Results indicate that ACE2 null mice exhibit normal phenotype regarding MAP and cardiac function.

\subsection{Cardiac Function and Blood Pressure Post Ang II infusion}

The effect of chronic high dose Ang II infusion (1000 ng/kg/min for 28 days) was examined on Echo and BP parameters for both groups (Figure 5). Ang II produced a marked reduction in FS\% in ACE2 KO, from $40 \%$ to $27 \%$ (baseline vs. Ang II) (Figure 6). LVEDd was comparable between groups. However, LVESd and LVESa were significantly increased in Ang II ACE2 KO as compared to Ang II WT (Figures 5; a,b). The end result was a reduction in EF\% of almost $31 \%$ in Ang II ACE2 KO as compared to $12 \%$ (NS) in Ang II WT (Figure 7). Results from the PWD mode showed a lower E/A ratio in Ang II ACE2 KO which suggests a diastolic dysfunction (Figure 8). Chronic Ang II infusion increased MAP with equal pressor responses in the groups (maximal increase of $\sim 40 \mathrm{mmHg}$, Figure 9). Notably, HR was not changed during serial Echo measurements 
in both strains (Figure 10). Results suggest a cardioprotective role of ACE2 against the systolic and diastolic dysfunction induced by Ang II infusion.

\subsection{Cardiomyocyte Size Quantitation}

Cardiac cell size was measured in H\&E stained tissue using computerized analytical methods. Microscopic examination of the cardiomyocytes within the left ventricle showed cellular expansion with shrinkage in the intercellular space in the Ang II ACE2 KO (Figure 11; a,b). Cell size quantitation within the left ventricular wall of Ang II ACE2 KO showed remarkably larger cardiomyocyte size than in Ang II WT (Figure 11c). This indicates cellular hypertrophy in the ACE2 KO. Results are compatible with cardiac hypertrophy observed in Ang II ACE2 KO determined by heart to body weight ratio (Figure 12; a,b). No differences were seen in the number of counted cells within the selected areas in the groups. Collectively, data suggest a defense mechanism of ACE2 against Ang II induce hypertrophic cardiomyopathy.

\subsection{Evidence for Cardiac and Aortic Fibrosis in Ang II ACE2 KO}

\subsubsection{Masson's Trichrome Staining for Collagen}

Replacement of myocytes with collagenous scar tissue is a major indicator of cardiac remodeling. Histological observation of Trichrome stained heart sections showed more myocardial collagen in Ang II ACE2 KO than in Ang II WT (Figure 13). The greatest collagen density was localized in the left ventricular wall.

\subsubsection{Picrosirius Red Staining for Collagen Fibers}


The collagen contents within the myocardium and aortic tissues were visualized using polarized light imaging of Picrosirius Red stained sections. This type of staining is selective for collagen Type I (yellow/orange fluorescence) and Type III (green fluorescence). Advantages of the method are high sensitivity, ability to detect thin collagen fibers, and to quantify collagen content. Heart and aortic sections were stained and images taken using external polarized light. Microscopic observation of myocardium showed higher left ventricular collagen content in Ang II ACE2 KO than in Ang II WT (Figures 14), (Figure 15). Analysis of collagen content revealed higher (CVF \%) in Ang II ACE2 KO compared to Ang II WT (Figure 16). Results are consistent to those observed with Trichrome staining (Figure 13).

Examination of the upper ascending thoracic aorta showed a similar enhancement in aortic remodeling as seen in the heart. There were marked differences in aortic structure and collagen content in Ang II ACE2 KO. Staining with Picrosirius Red revealed a dense concentration of collagen fibers in the aortic adventitia of Ang II ACE2 KO (Figure 17). Quantification showed a higher percent collagen in Ang II ACE2 KO (Figure 18) associated with a narrowing in the luminal vessel area (Figure 19). The high collagen content in the outermost layer along with the narrowing area of the aorta would indicate vessel stiffness and loss of compliance and elasticity. These changes suggest a greater cardiac afterload in Ang II ACE2 KO which compromises systolic function and negatively affects EF\%.

\subsection{DHE Staining for Superoxide}


Tests were performed to evaluate superoxide $\left(\mathrm{O}^{-}\right)$production in fresh frozen heart and aorta sections using DHE fluorescence staining as the marker. DHE is an efficient method to detect in-situ ROS formation (Peshavariya, Dusting, \& Selemidis, 2007) and it is very selective to $\mathrm{O}^{-}$production (Benov, Sztejnberg, \& Fridovich, 1998). Results showed that Ang II produced a marked effect on $\mathrm{O}^{-}$production in hearts of ACE2 KO (Figures 20). Results were quantified according to the intensity of ROS pixels expressed as ROS\% to the total section pixels. Myocardial sections from Ang II ACE2 KO exhibited higher ROS\% compared to Ang II WT (Figure 21). Microscopic examination of aorta sections stained with DHE also revealed higher $\mathrm{O}^{-}$levels in Ang II ACE2 KO as compared to Ang II WT (Figures 22). Aorta from Ang II ACE2 KO showed higher ROS\% than Ang II WT (Figures 23). Results suggest that ACE2 may act as a scavenger against tissue superoxide production. 
Table 1. Detailed Echo data analysis of cardiac function in the WT and ACE2 KO at the baseline and after 4 weeks of Ang II stimulation.

\begin{tabular}{|c|c|c|c|c|}
\hline & \multicolumn{2}{|c|}{ Baseline } & \multicolumn{2}{c|}{ Treatment } \\
\hline NT & N=8 & NCE2 KO & Ang II WT & $\begin{array}{c}\text { Ang II ACE2 } \\
\text { KO }\end{array}$ \\
\hline HR(bpm) & $410 \pm 18$ & $436 \pm 22$ & N=7 & N=7 \\
\hline LVEDa (cm $)$ & $0.190 \pm 0.004$ & $0.199 \pm 0.004$ & $0.191 \pm 0.006$ & $0.208 \pm 0.006$ \\
\hline LVEDd (cm) & $0.376 \pm 0.007$ & $0.40 \pm 0.007$ & $0.392 \pm 0.009$ & $0.398 \pm 0.009$ \\
\hline LVESa (cm $)$ & $0.129 \pm 0.005$ & $0.139 \pm 0.005$ & $0.170 \pm 0.006 \dagger$ & $0.198 \pm 0.006 \dagger^{*}$ \\
\hline LVESd (cm) & $0.226 \pm 0.007$ & $0.242 \pm 0.007$ & $0.244 \pm 0.006$ & $0.284 \pm 0.006 \dagger^{*}$ \\
\hline FS (\%) & $40.27 \pm 0.8$ & $39.64 \pm 0.79$ & $37.85 \pm 0.37$ & $27.46 \pm 0.36 \dagger^{*}$ \\
\hline SWT (cm) & $0.068 \pm 0.002$ & $0.067 \pm 0.002$ & $0.071 \pm 0.0037$ & $0.076 \pm 0.003$ \\
\hline PWT (cm) & $0.072 \pm 0.003$ & $0.067 \pm 0.002$ & $0.082 \pm 0.002$ & $0.091 \pm 0.002 \dagger$ \\
\hline RWT & $0.381 \pm 0.016$ & $0.346 \pm 0.015$ & $0.394 \pm 0.024$ & $0.423 \pm 0.024 \dagger$ \\
\hline EF (\%) & $57.8 \pm 3.3$ & $57.27 \pm 3.35$ & $50.35 \pm 1.11$ & $39.52 \pm 1.12 \dagger^{*}$ \\
\hline E-wave (cm/s) & $69.97 \pm 2.4$ & $72.94 \pm 2.4$ & $55.14 \pm 1.65 \dagger$ & $52.08 \pm 1.65 \dagger$ \\
\hline A-wave (cm/s) & $38.8 \pm 1.44$ & $35.57 \pm 1.45$ & $40.64 \pm 1.0$ & $40.11 \pm 0.97$ \\
\hline E/A Ratio & $1.83 \pm 0.025$ & $1.80 \pm 0.025$ & $1.65 \pm 0.011 \dagger$ & $1.29 \pm 0.012 \dagger^{*}$ \\
\hline IVRT (ms) & $15.64 \pm 0.7$ & $14.95 \pm 0.71$ & $18.07 \pm 0.5$ & $19.74 \pm 0.47 \dagger$ \\
\hline IVCT (ms) & $19.06 \pm 0.76$ & $17.96 \pm 0.7$ & $20.9 \pm 0.68$ & $21.58 \pm 0.7 \dagger$ \\
\hline
\end{tabular}

Data were analyzed using repeated measure 2 way ANOVA. Modified Tukey post hoc test was used to compare means; ${ }^{*} p<0.05$ vs. WT; $\uparrow p<0.05$ vs. baseline. HR, heart rate; LVEDa, left ventricular end diastolic area; LVEDd, left ventricular end diastolic diameter; LVESa, left ventricular end systolic area; LVESd, left ventricular end systolic diameter; FS\%, fractional shortening; SWT, septal wall thickness; PWT, posterior wall 
thickness; RWT, relative wall thickness; EF\%, ejection fraction; E-wave, peak early transmitral inflow mitral E velocity; A-wave, transmitral inflow velocity due to atrial contraction. 

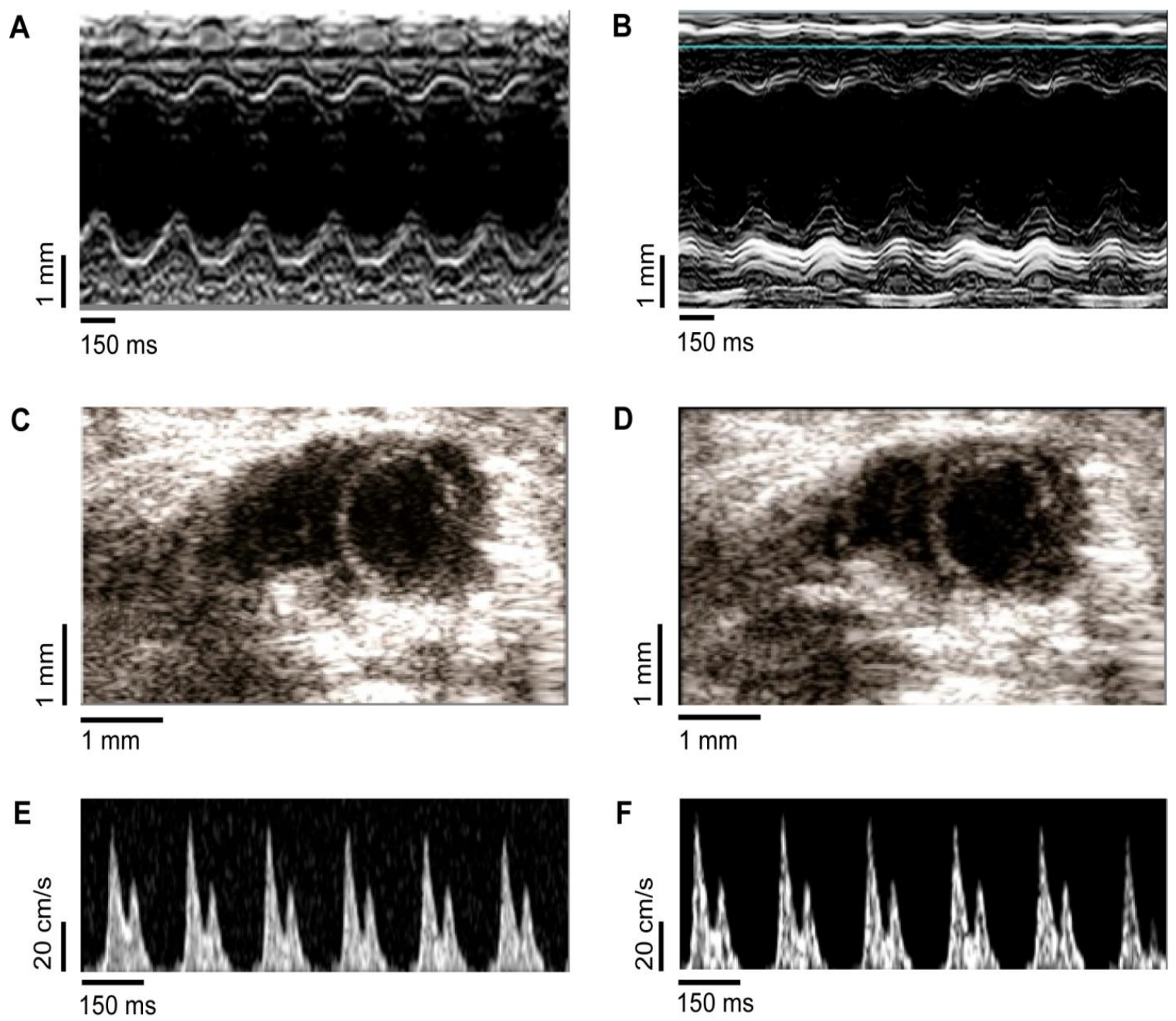

Figure 4: Baseline Echocardiography images of LV in WT (A) and ACE2 KO (B).

Representative 2D M-Mode images of LV show similar dimensions in the groups.

Parasternal short axis 2-D images shows similar LV end systolic area (LVESa) in WT (C) and ACE2 KO (D). Images were standardized by the aortic annulus and the papillary muscles during the long axis and short axis views, respectively. Transmitral pulse wave Doppler mode shows similar mitral valve function in WT (E) and ACE2 KO (F). 

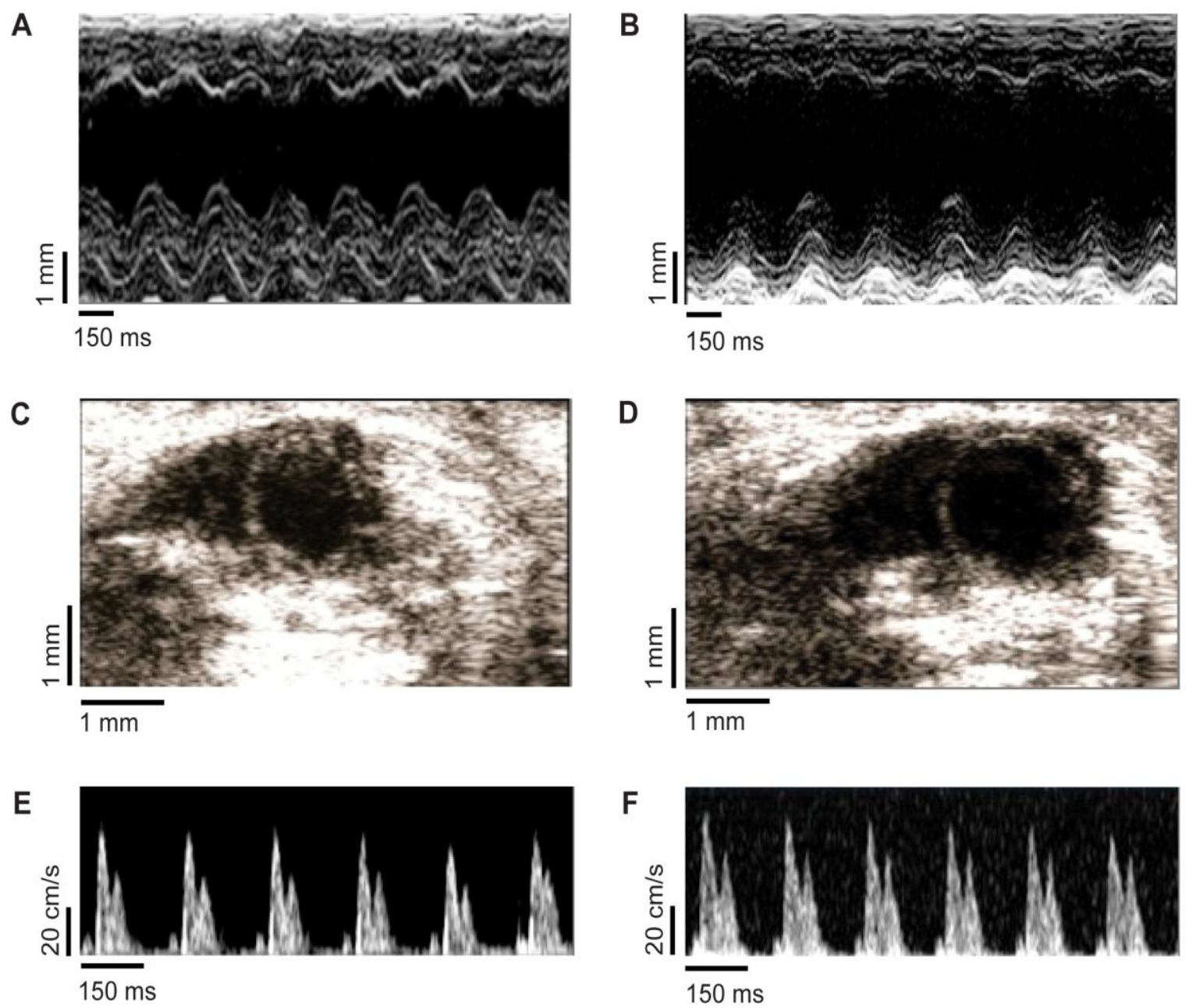

Figure 5: Echocardiographic assessments after 4 weeks of Ang II infusion.

Representative 2D M-Mode images of LV show an increase in LVESd in ACE2 KO (B) as compared to WT (A). Parasternal short axis 2-D images shows an exacerbated LV end systolic area (LVESa) in ACE2 KO (D) as compared to WT (C). Images were standardized by the aortic annulus and the papillary muscles during the long axis and short axis views, respectively. Transmitral pulse wave doppler mode shows a decrease in E-wave velocity in both groups WT (E), ACE2 KO (F) compared to their corresponding baseline images. 


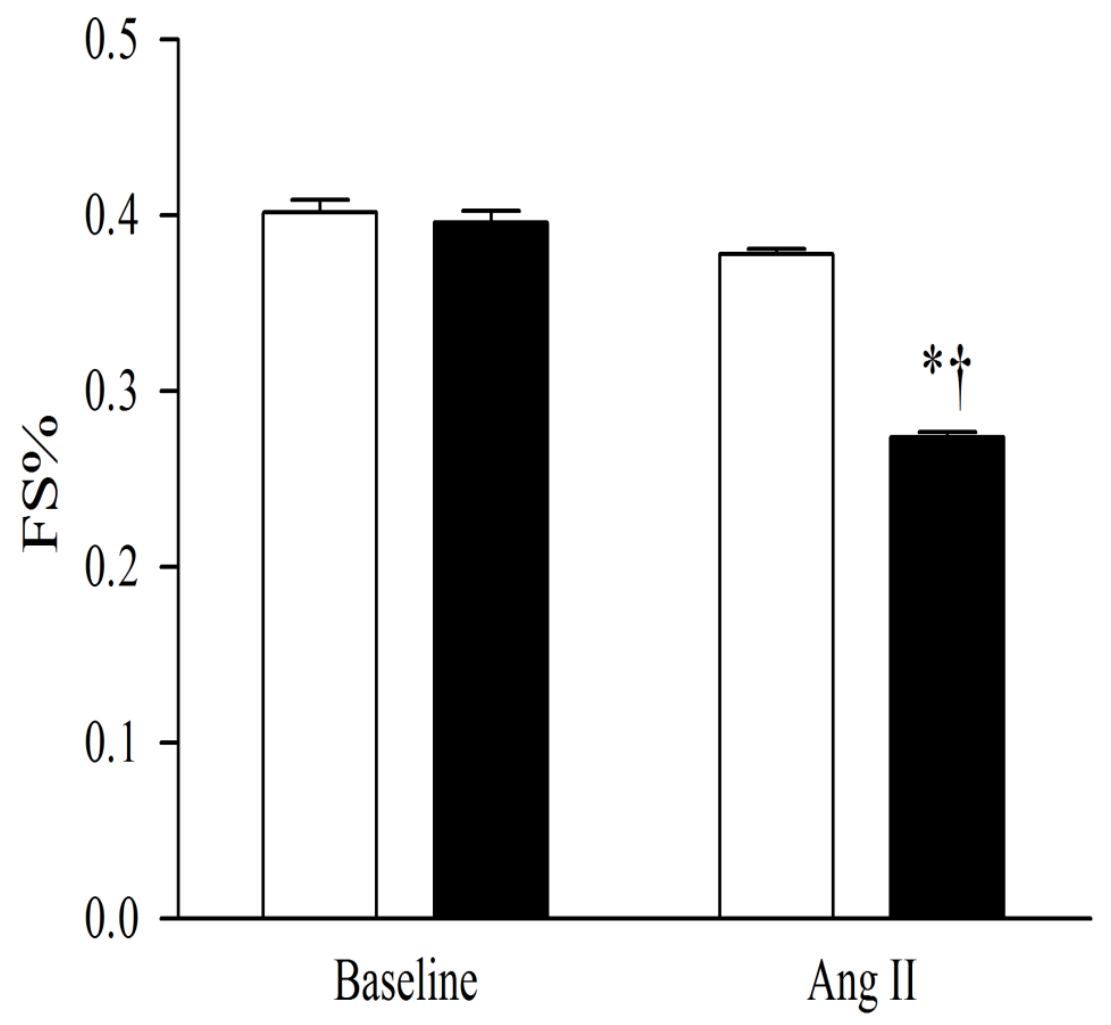

Figure 6: Percent fractional shortening (FS\%) at baseline and after 4 weeks of Ang II infusion. Results showed decrease in FS\% in Ang II ACE2 KO compared to Ang II WT and baseline. Data were analyzed using repeated measure 2 way ANOVA. Modified Tukey post hoc test was used to compare means; ${ }^{*} p<0.05$ vs. WT; $\dagger p<0.05$ vs. baseline. Data are represented as mean \pm SEM of group size $(n=7-8)$. 


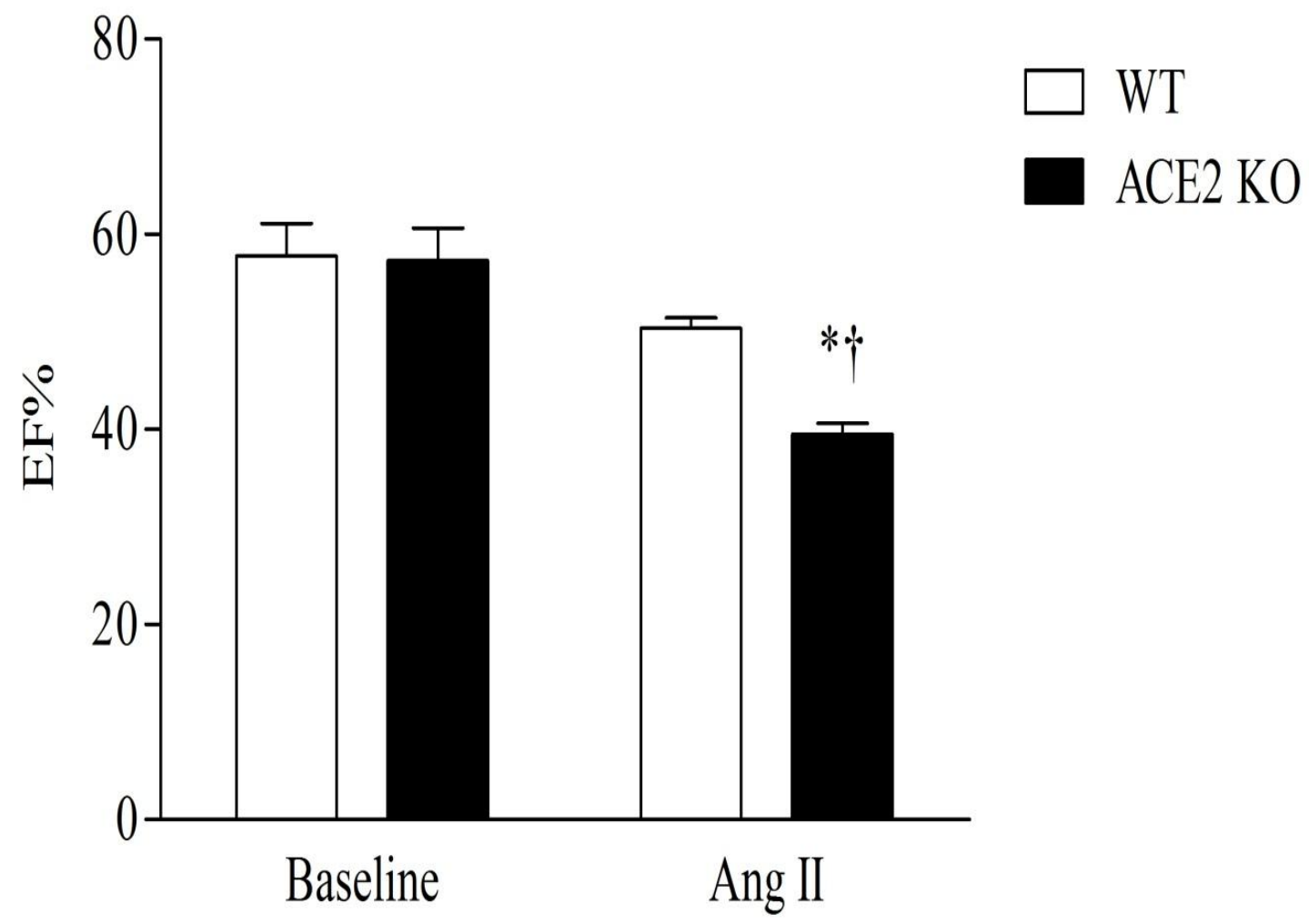

Figure 7: Percent ejection fraction (EF\%) at baseline and after 4 weeks of Ang II infusion. Results showed decrease in EF\% in Ang II ACE2 KO compared to Ang II WT and baseline. Data were analyzed using repeated measure 2 way ANOVA. Modified Tukey post hoc test was used to compare means; ${ }^{*} p<0.05$ vs. WT; $\uparrow p<0.05$ vs. baseline. Data are represented as mean \pm SEM of group size $(n=7-8)$. 


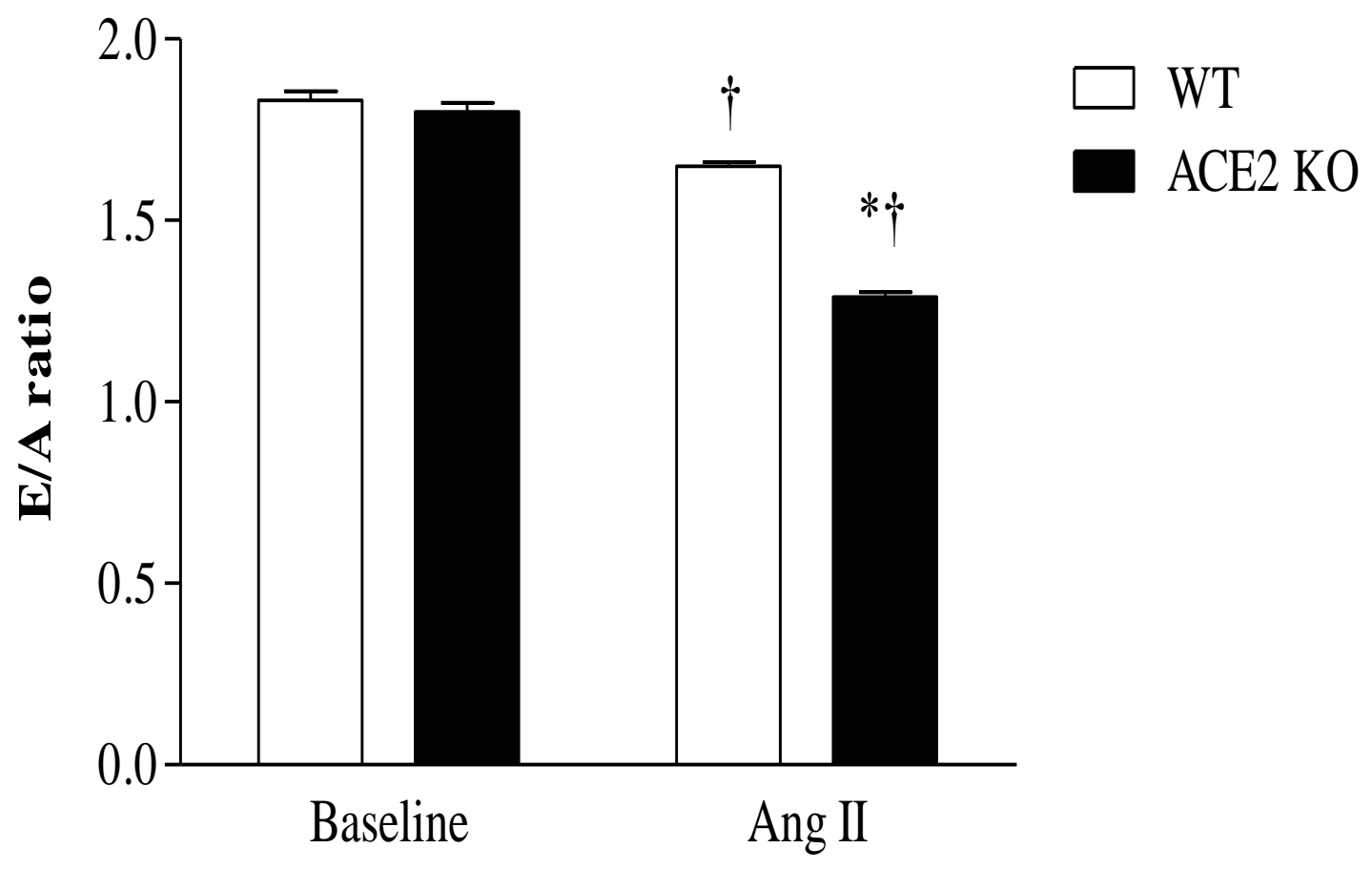

Figure 8: E/A ratio at baseline and after 4 weeks of Ang II infusion. Results showed a decrease in E/A ratio in Ang II ACE2 KO compared to Ang II WT and baseline. Data were analyzed using repeated measure 2 way ANOVA. Modified Tukey post hoc test was used to compare means; ${ }^{*} p<0.05$ vs. WT; $\dagger p<0.05$ vs. baseline. Data are represented as mean \pm SEM of group size $(n=7-8)$. 


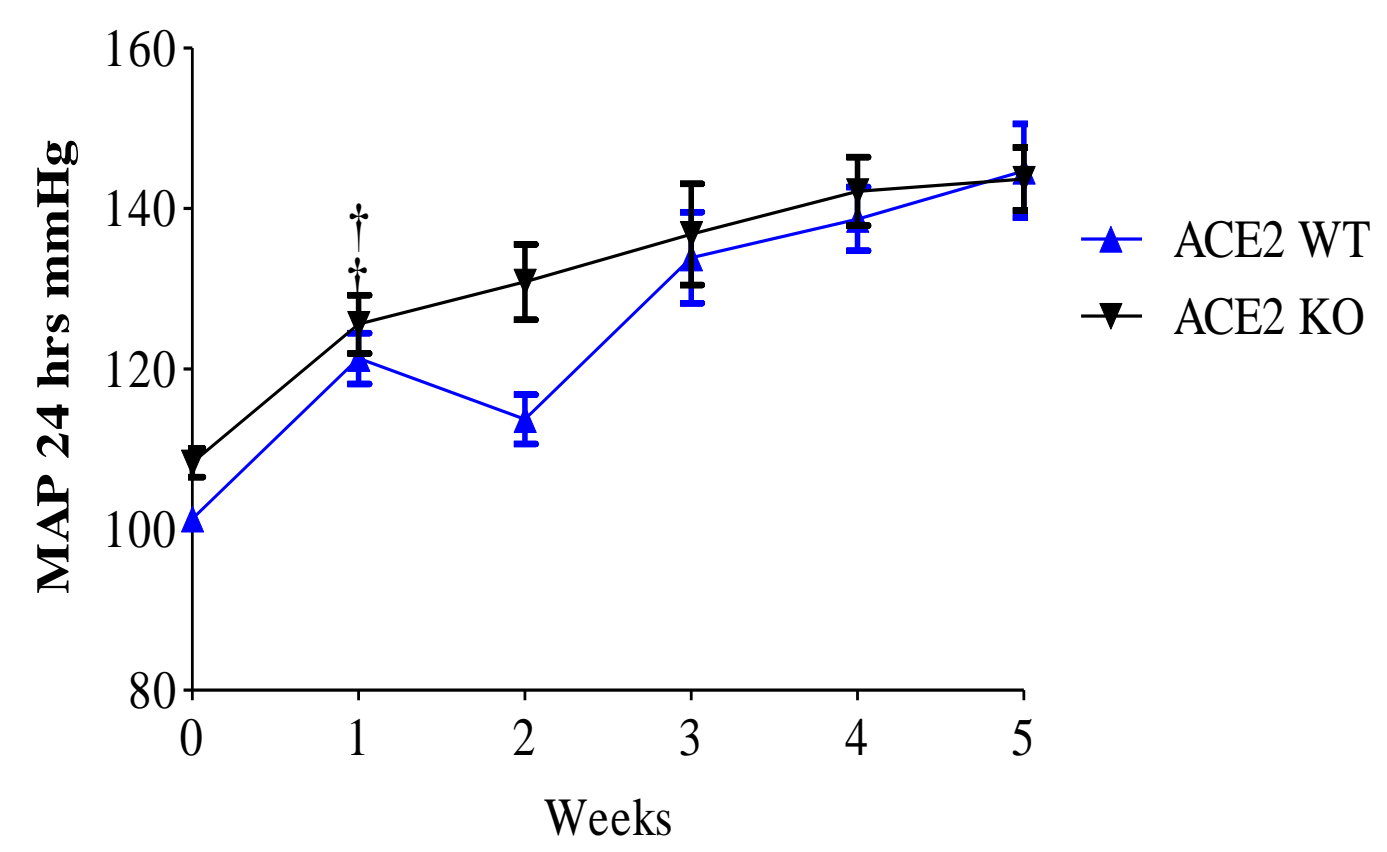

Figure 9: Mean arterial pressure (MAP) in Ang II infused WT and ACE2KO. MAP was recorded for 24 hours. Results showed a significant increase in MAP after the first week of Ang II infusion with no significant between strains. Data were analyzed using repeated measure 2 way ANOVA. Modified Tukey post hoc test was used to compare means; $\dagger p<0.05$ vs. baseline. Data are represented as mean \pm SEM of group size $(\mathrm{n}=7-8)$. 


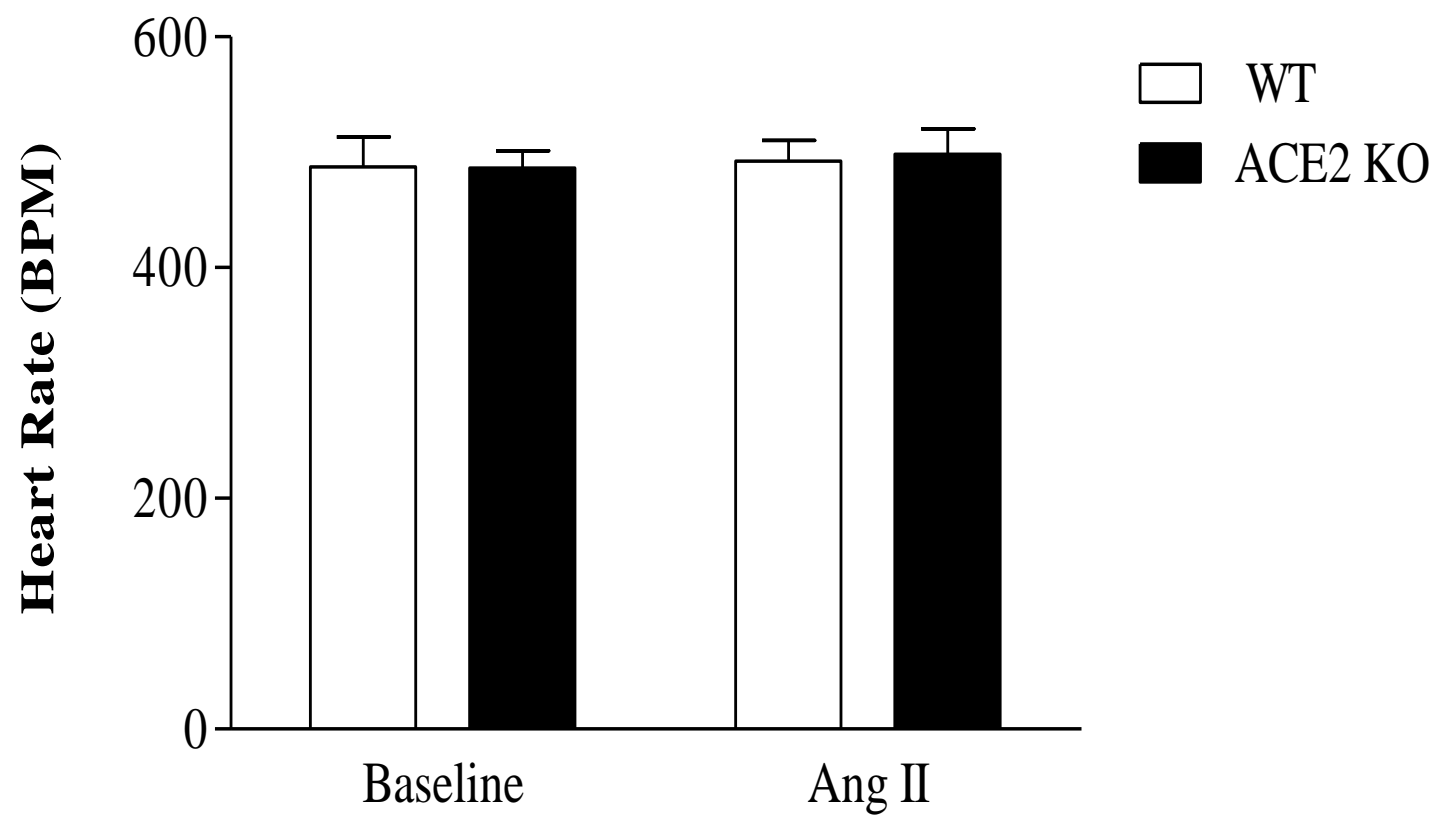

Figure 10: Heart rate (HR) in WT and ACE2 KO. Data was recorded at the baseline and after 4 weeks of Ang II infusion. No difference was observed between both strain before and after treatment. Data were analyzed using repeated measure 2 way ANOVA.

Modified Tukey post hoc test was used to compare means. Data are represented as mean \pm SEM of group size $(n=7-8)$. 

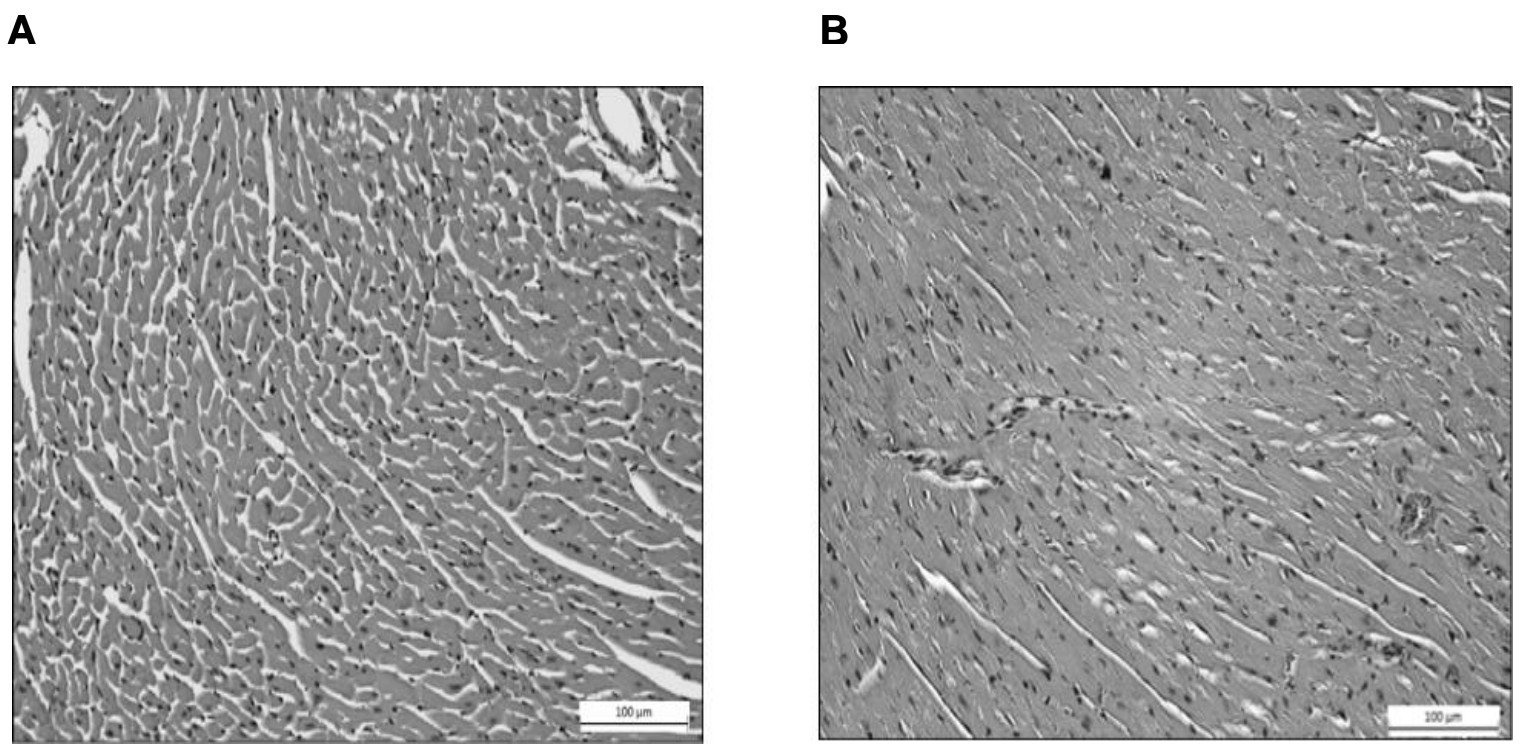

C

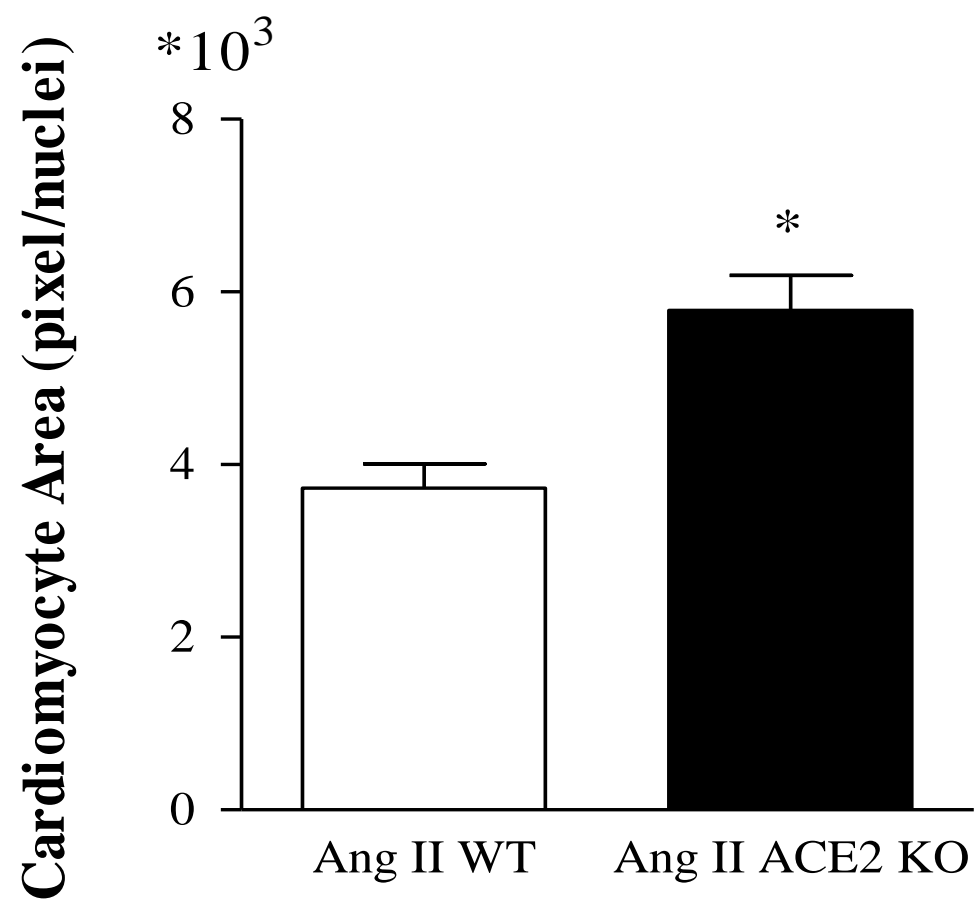


Figure 11: H\&E stained sections within LV wall of Ang II infused WT (A) and Ang II infused ACE2 KO (B). Greyscale images were taken at (20X) magnification for quantitation purpose. Histological examination showed an increase in the cardiomyocyte area with shrinkage in the intercellular space. Image software was used to normalize and threshold the images as discussed in the methods section. Cardiomyocyte size was computed as (pixel/nuclei) (C). Cell size quantitation shows larger cardiomyocytes area in the LV in Ang II infused ACE2 KO vs. Ang II infused WT. Data were analyzed using Unpaired student t-test; $* p<0.05$ vs. Ang II WT. Data are represented as mean \pm SEM. 
A

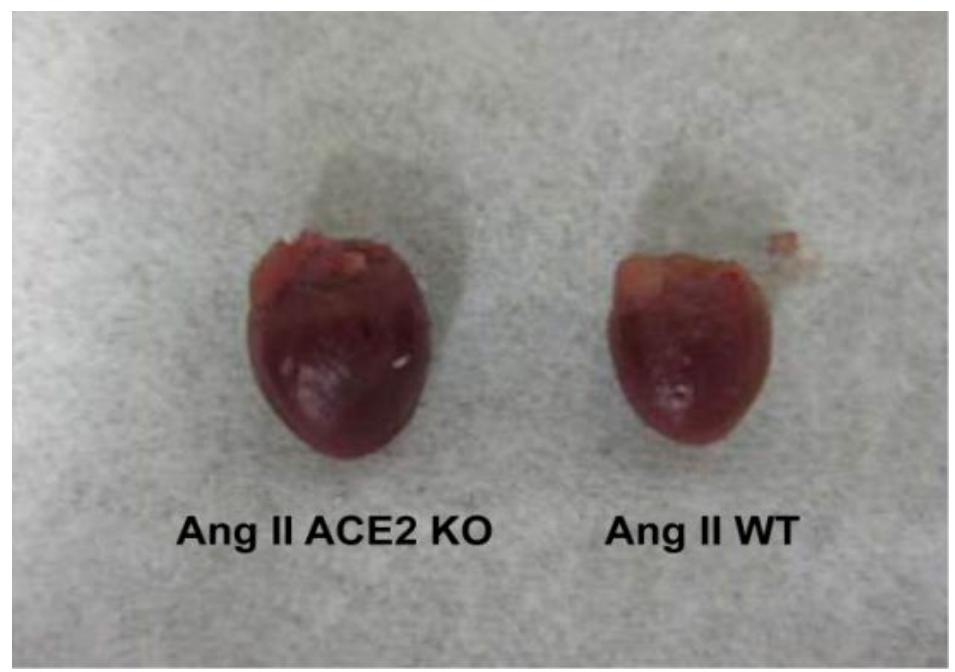

B

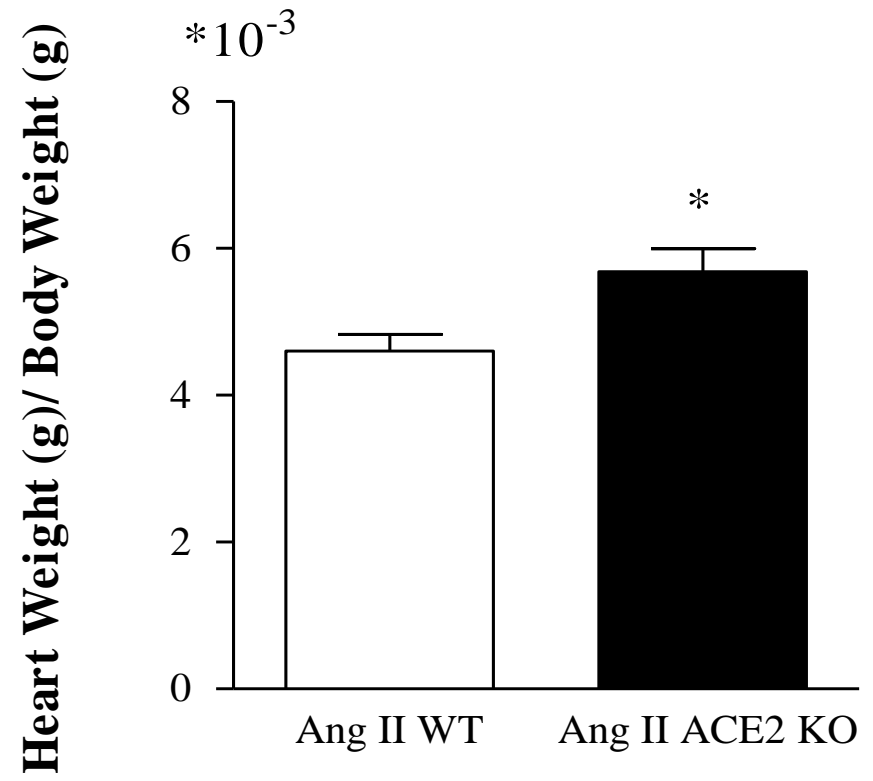


Figure 12: Heart weight/Body weight was computed for both groups after Ang II infusion. Results showed higher ratio in ACE2 KO group compared to WT. Data were analyzed using Unpaired student t-test; ${ }^{*} p<0.05$ vs. Ang II WT. Data are represented as mean \pm SEM. 
A

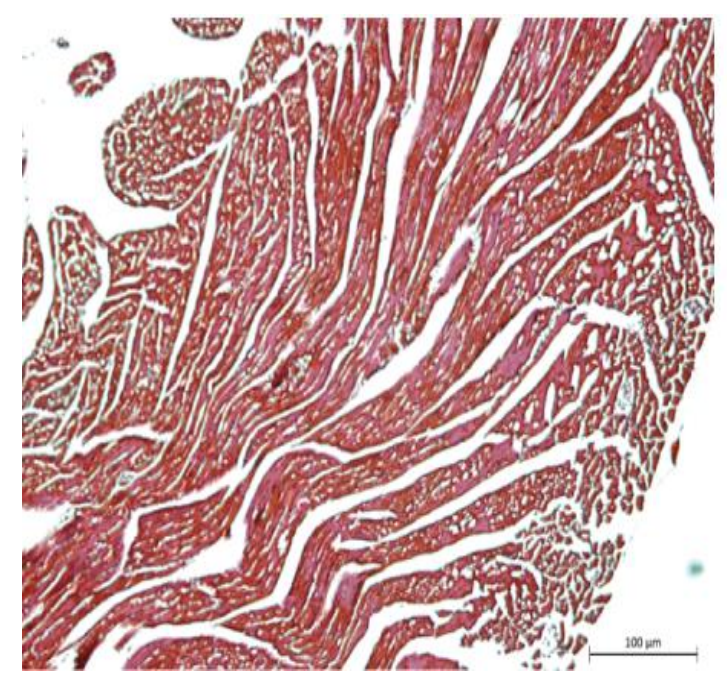

C

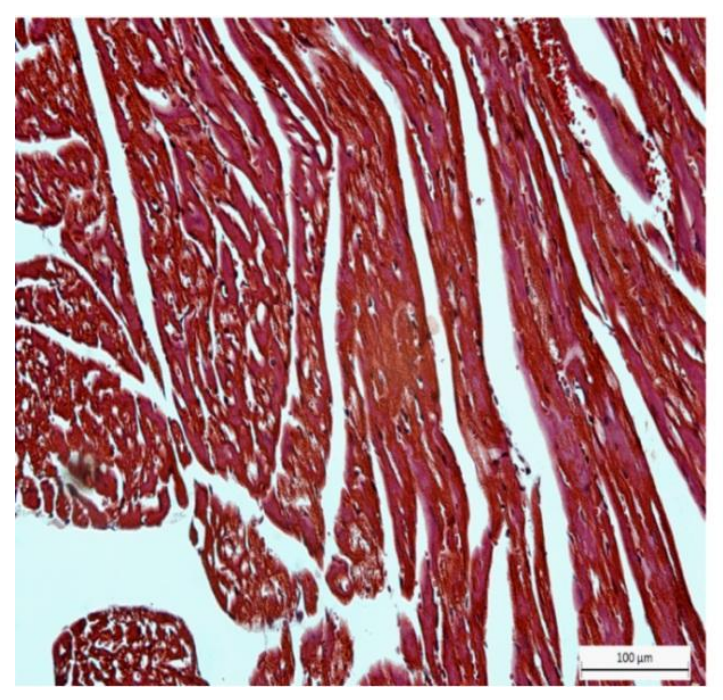

B

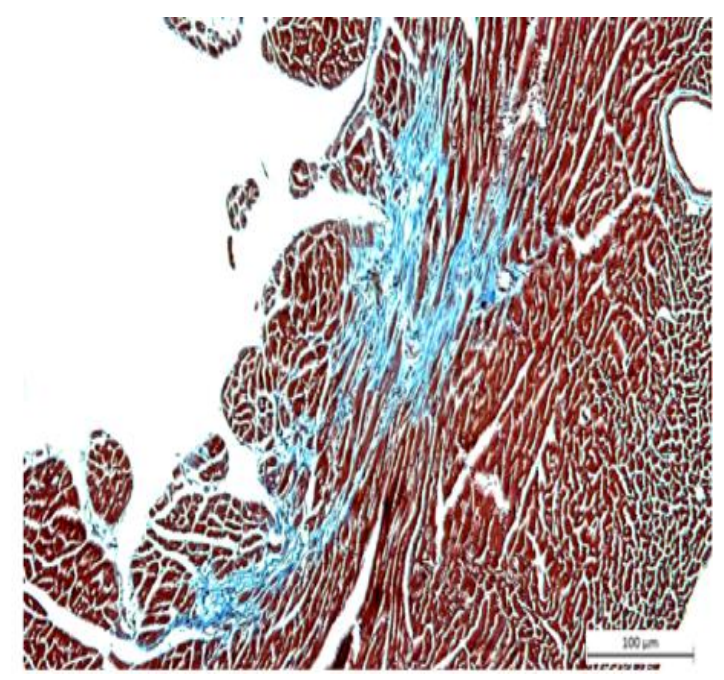

D

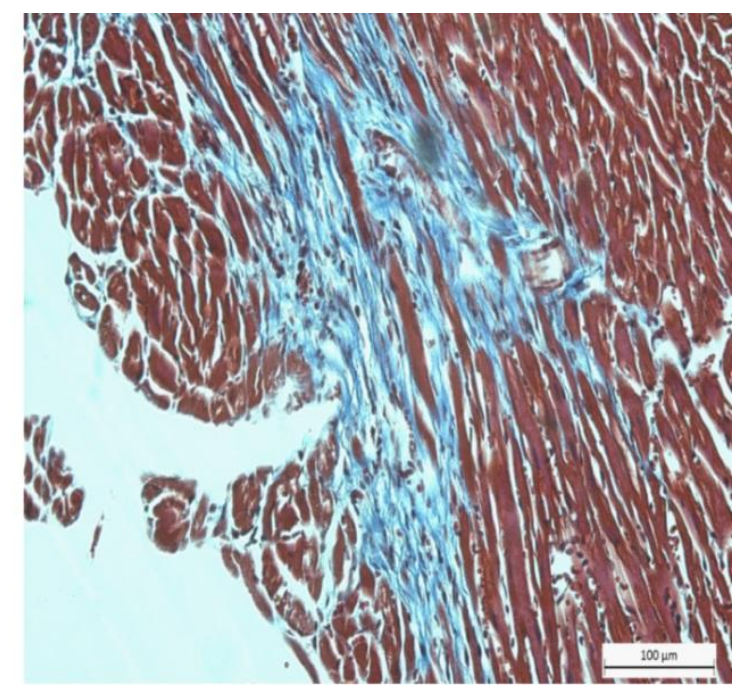

Figure 13: Masson's Trichrome staining for collagen in myocardial sections in Ang II WT (A),(C) and Ang II ACE2 KO (B),(D), at (10X), (20X) magnification, respectively. Collagen content (blue) was seen within the interstitial space of Ang II ACE2 KO indicating myocardial remodeling. 
A

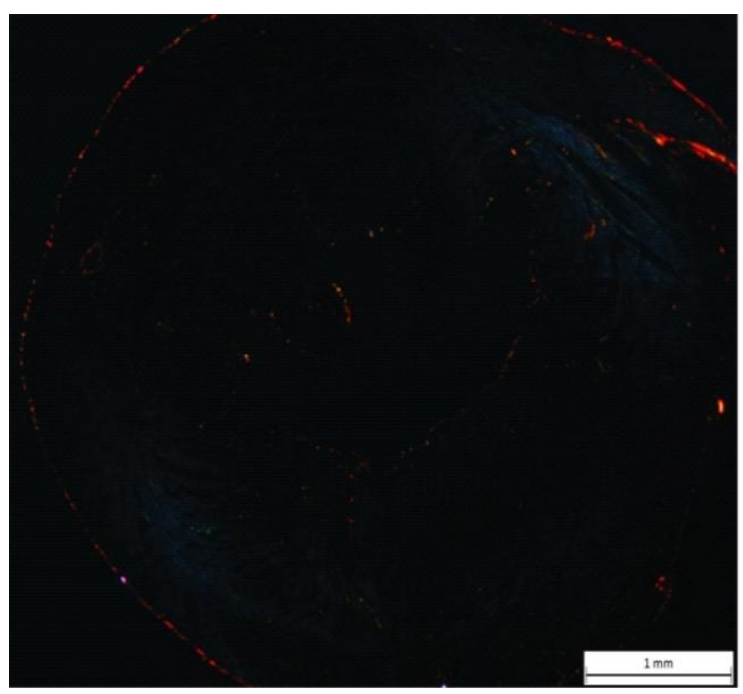

B

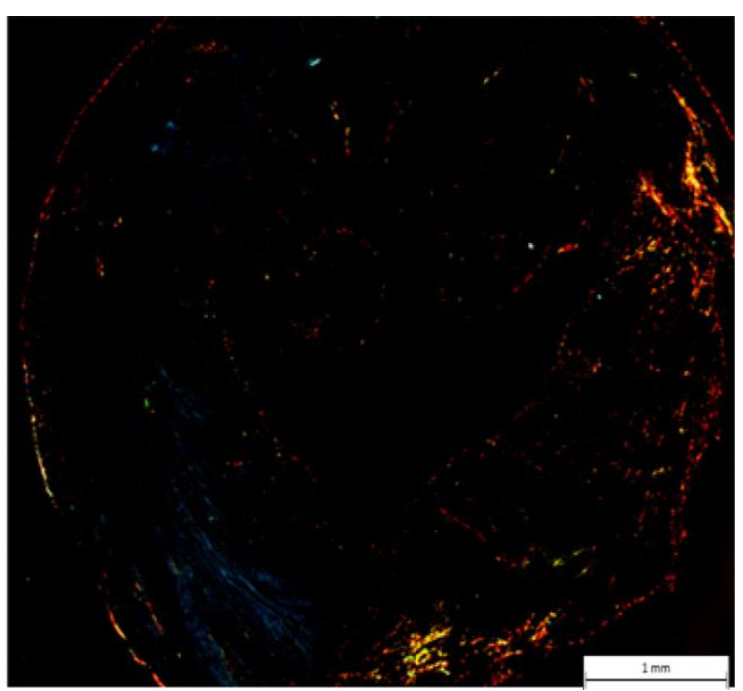

Figure 14: Myocardium sections stained for collagen using Picrosirius Red in Ang II WT (A) and Ang II ACE2 KO (B). Tissues were examined using polarized light. Type I collagen fibers are highly birefringent, yellow or orange glow. Histological examinations showed an overt higher collagen content within the myocardium of ACE2 KO compared to WT. Images were taken at (2.5X) magnification for quantitation purpose. 
A

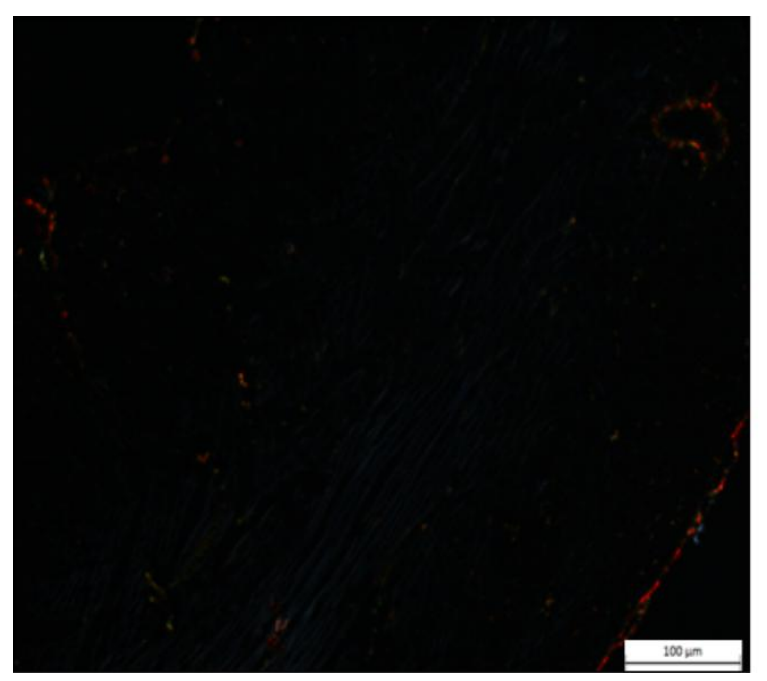

B

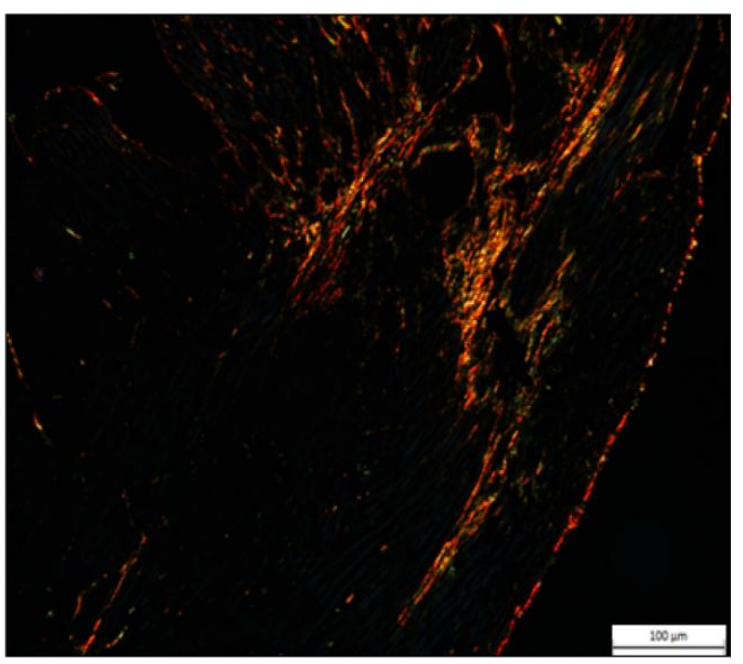

Figure 15: Myocardium sections stained for collagen using Picrosirius Red in Ang II WT (A) and Ang II ACE2 KO (B). Tissues were examined using polarized light. Type I collagen fibers are highly birefringent, yellow or orange glow. Histological examinations showed an overt higher collagen content within the myocardium of ACE2 KO compared to WT. Images were taken at (20X) magnification. 


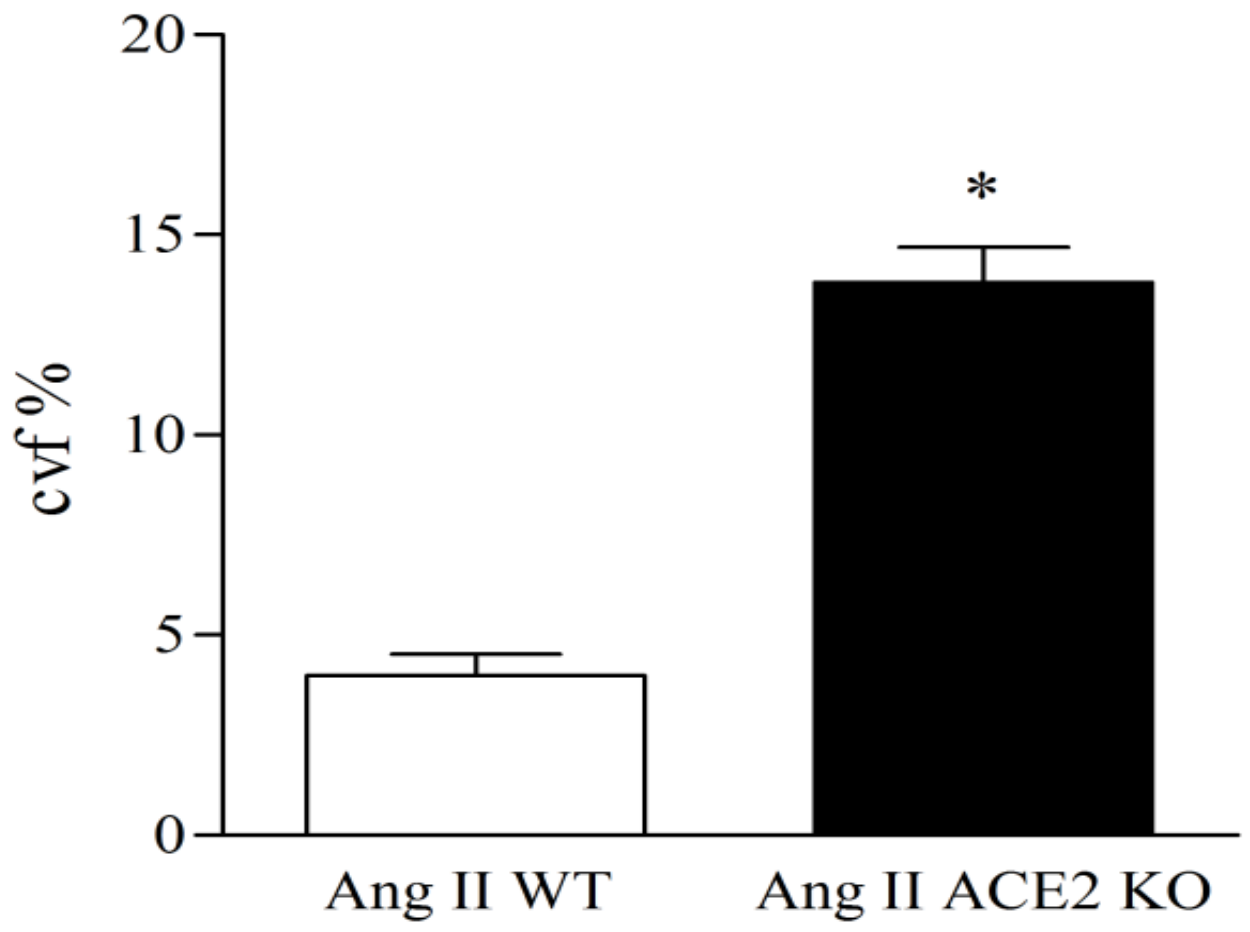

Figure 16: Quantitation of myocardial collagen contents of Ang II WT and Ang II ACE2 KO. Results showed higher collagen volume fraction (CVF\%) in Ang II ACE2 KO compared to WT. Analysis was done using Metamorph ${ }^{\circledR} 7.6 .3$ image analysis software as described in the method section. Data were analyzed using Unpaired student t-test; ${ }^{*} p<0.05$ vs. Ang II WT. Data are represented as mean \pm SEM. 
A

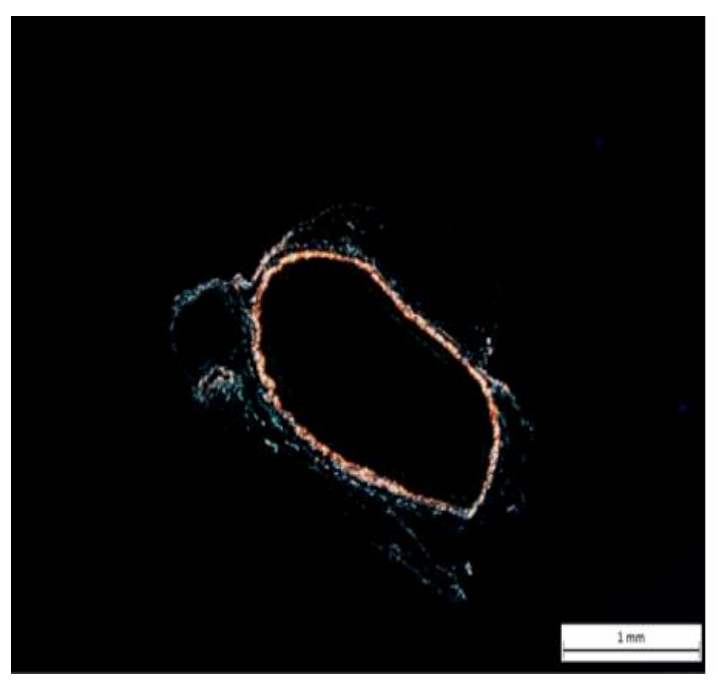

B

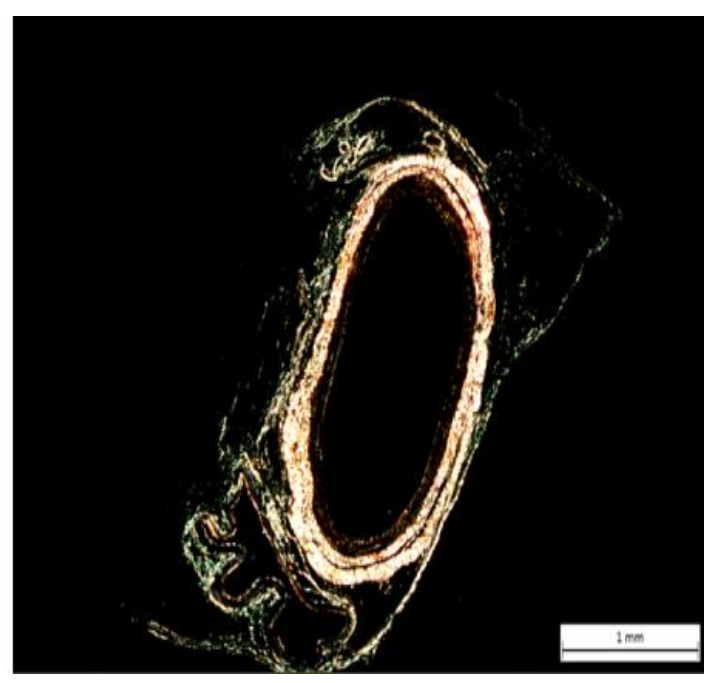

Figure 17: Sections of aortas were stained with Picrosirius Red in Ang II WT (A) and Ang II ACE2 KO (B). Images were taken in polarized light at (2.5X) magnification. Collagen fibers were quite dominant within the adventitia layer of Ang II ACE2 KO. 


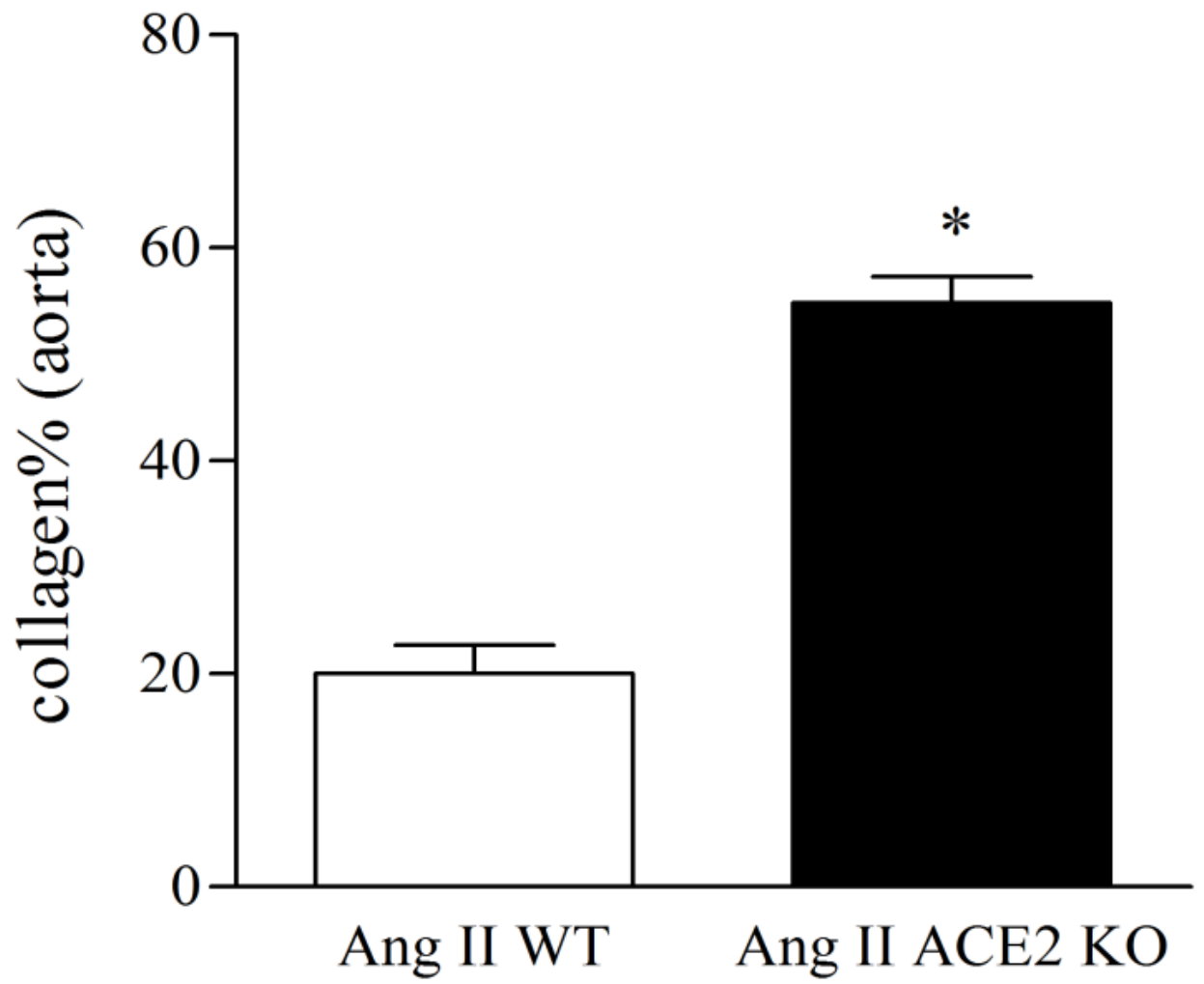

Figure 18: Quantitation of aortic collagen contents of Ang II WT and Ang II ACE2 KO. Results shows that collagen percentage (collagen \%) was more than two fold higher in Ang II ACE2 KO as compared to Ang II WT. Analysis was done using Metamorph ${ }^{\circledR}$ 7.6.3 image analysis software as described in the method section. Data were analyzed using Unpaired student t-test; ${ }^{*} p<0.05$ vs. Ang II WT. Data are represented as mean \pm SEM. 


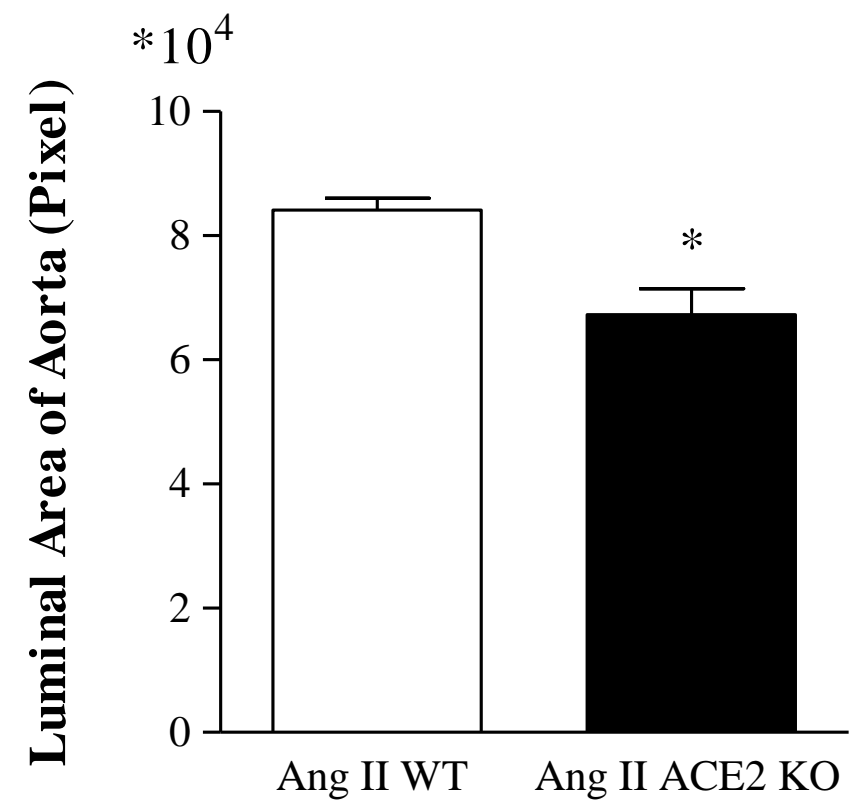

Figure 19: Quantitation of the luminal area of the aorta showed higher surface area (pixels) in Ang II WT than in Ang II ACE2 KO suggesting an aortic stenosis in the ACE2 KO mice. Area measurements were done using Metamorph ® 7.6.3 image analysis software as described in the method section. Data were analyzed using Unpaired student t-test; $* p<0.05$ vs. Ang II WT. Data are represented as mean \pm SEM. 
A

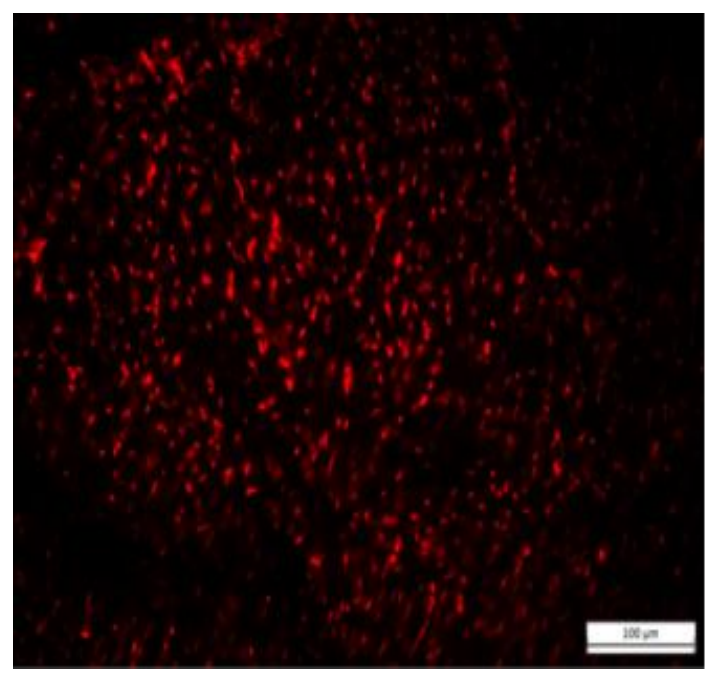

B

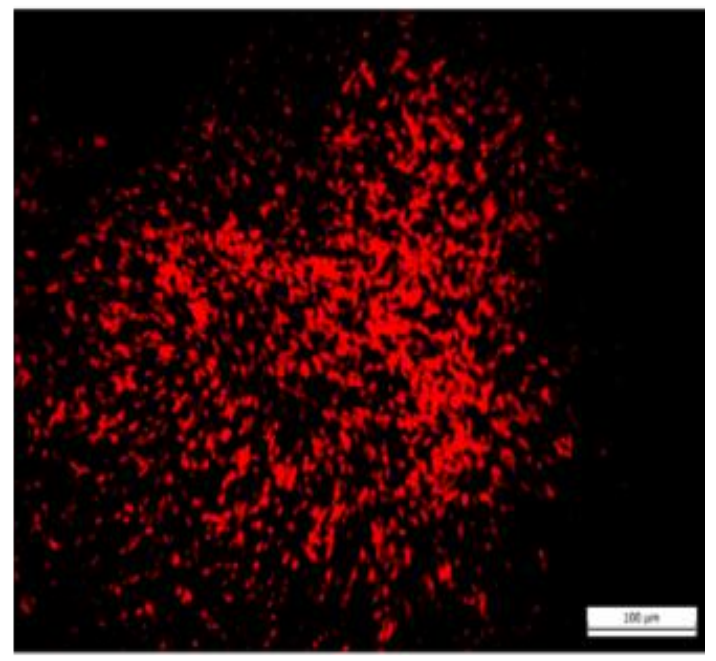

Figure 20: Dihydroethidium (DHE) staining of myocardial sections of Ang II infused mice: WT (A) and ACE2 KO (B). Results showed a higher superoxide production within the myocardium of Ang II ACE2 KO compared Ang II WT as indicated by DHE fluorescence. Images were taken at (20X) magnification. 


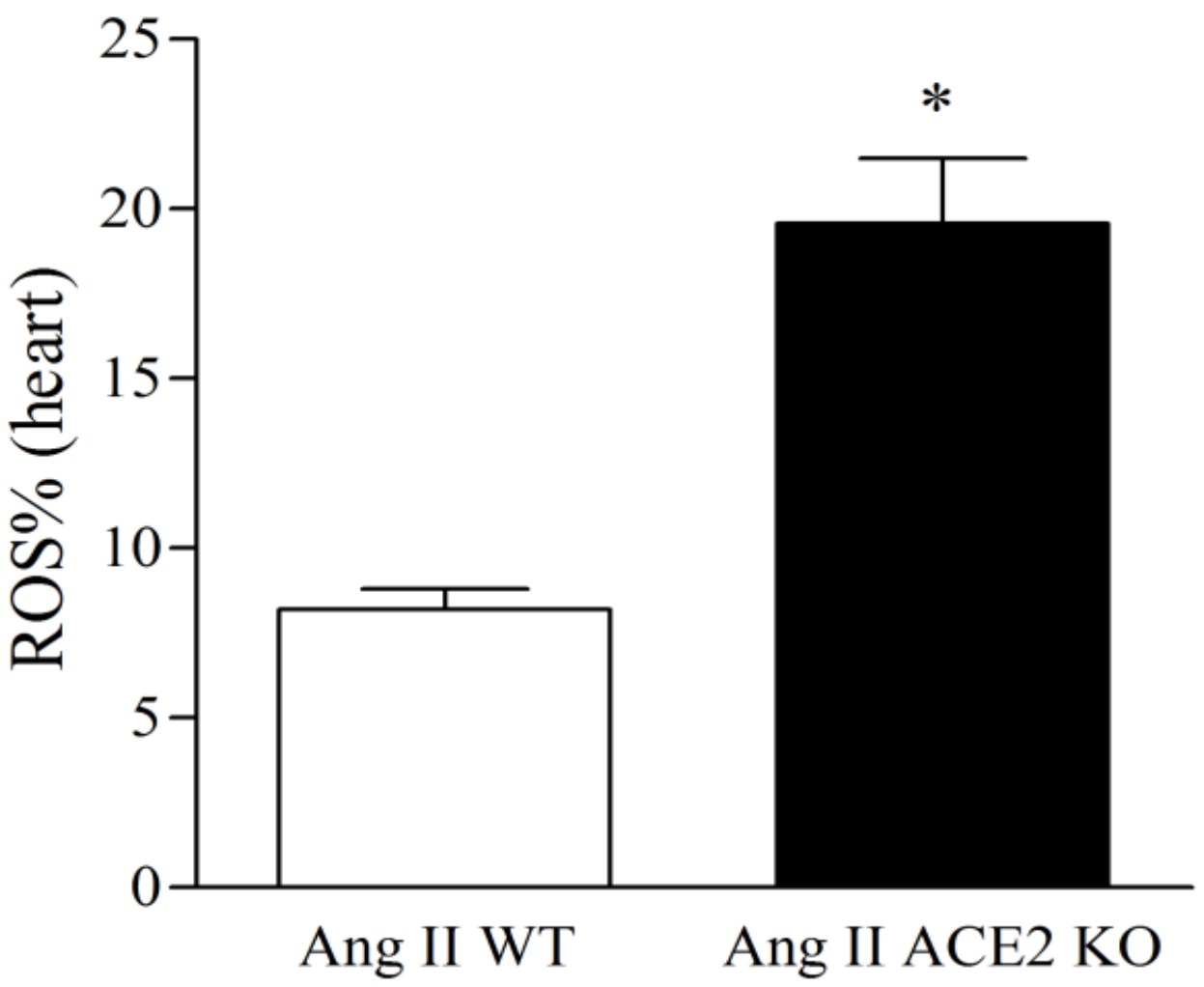

Figure 21: Quantitation of DHE stained myocardial section revealed a higher ROS\% as indicated by DHE within the myocardium of Ang II ACE2 KO. This suggests a protective role of ACE2 against Ang II induced myocardial oxidative stress. Analysis was done using Metamorph ${ }^{\circledR}$ 7.6.3 image analysis software as described in the method section. Data were analyzed using Unpaired student t-test; ${ }^{*} p<0.05$ vs. Ang II WT. Data are represented as mean $\pm \mathrm{SEM}$. 
A

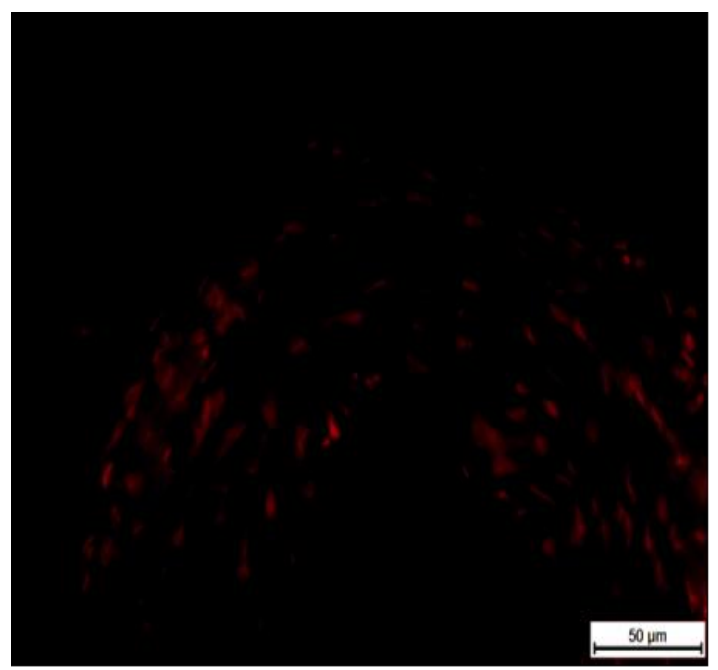

B

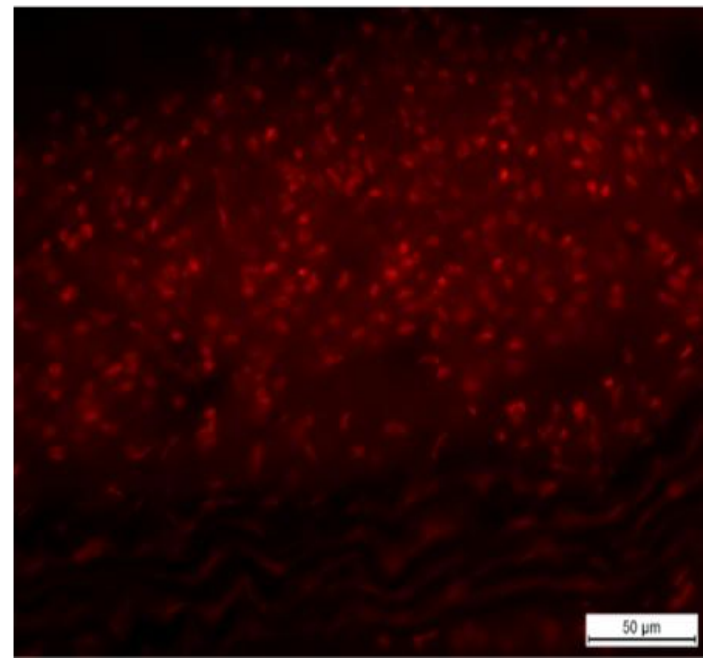

Figure 22: Dihydroethidium (DHE) staining of Aortas of Ang II infused mice: WT (A) and ACE2 KO (B). Results showed a higher superoxide production within the aorta of Ang II ACE2 KO compared Ang II WT as indicated by DHE fluorescence. 


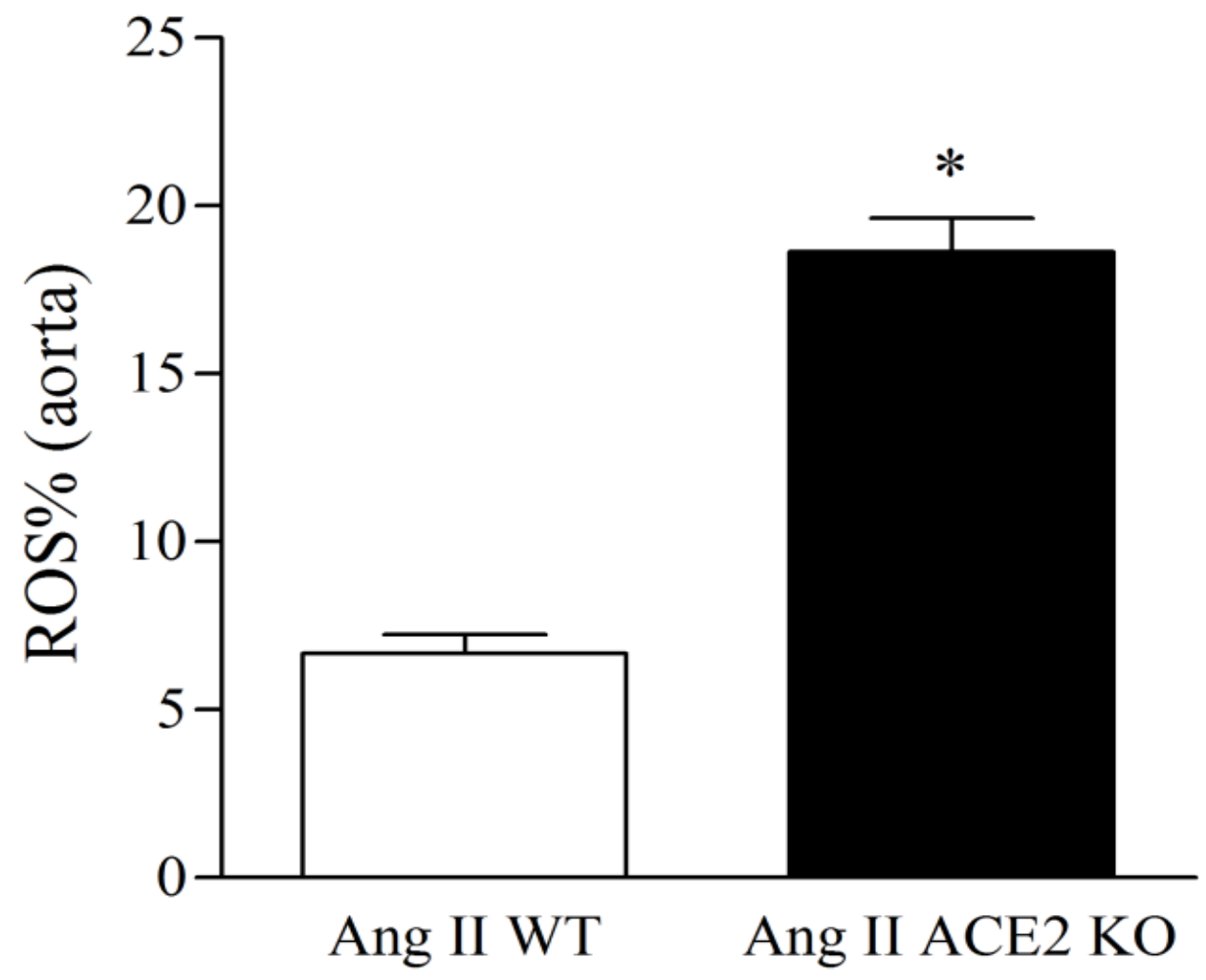

Figure 23: Quantitation of DHE stained aortic sections revealed a higher ROS\% within the aorta of Ang II ACE2 KO. Analysis was done using Metamorph® 7.6.3 image analysis software as described in the method section. Data were analyzed using Unpaired student t-test; $* p<0.05$ vs. Ang II WT. Data are represented as mean \pm SEM. 


\section{DISCUSSION}

ACE2 is a monocarboxypeptidase that functions beneficially to reduce the deleterious effects of Ang II by metabolizing it to Ang (1-7) (Donoghue et al., 2000; Elased, Cunha, Coffman, Gurley, \& Morris, 2005; Tipnis et al., 2000; Chappell, Allred, \& Ferrario, 2001). ACE2 has been detected in many organs (Harmer, Gilbert, Borman, \& Clark, 2002) although it is highly expressed in the heart (Donoghue et al., 2000; Tipnis et al., 2000). To explore ACE2's role in the development of Ang II induced cardiovascular pathologies, the ACE2 KO strain was used (Gurley et al., 2006). The objective was to establish the functional and structural outcomes in mice lacking ACE2 and to determine the effects of chronic Ang II infusion on BP (radiotelemetry), cardiac function (Echo), and cardiac/aortic structure (histology). At baseline conditions, 8 weeks old ACE2 KO mice exhibit normal cardiac function. This is consistent with results from Gurley et al. (2006) using ACE2 KO mice with the same genetic background, age, and gender. After chronic Ang II stimulation, there were a cadre of symptoms which document ACE2's cardioprotective role. Damage in the Ang II infused ACE2 KO was documented by increased cardiac systolic dimensions, overt hypertrophic cardiomyopathy, as well as

aortic remodeling. These changes were associated with enhanced oxidative stress seen in the heart and aorta. Collectively, findings point toward ACE2 as an essential modulator to the maladaptive cardiovascular response resulted from long term Ang II stimulation.

ACE2 was first cloned from the left ventricle of a patient with heart failure (Donoghue et al., 2000; Tipnis et al., 2000) and early studies suggested that ACE2 deletion produced cardiac contractile dysfunction (Crackower et al., 2002). However, this did not prove to 
be a universal finding since there were reports (Gurley et al., 2006; Yamamoto et al., 2006) that ACE2 KO mice with a mixed genetic background exhibited normal cardiac function at the same age. It is worth noting that the cardiac contractile dysfunction observed in some ACE2 KO models (Crackower et al., 2002; Oudit et al., 2007; Nakamura et al., 2008) might be explained by the methods used for ACE2 gene disruption and/or the genetic background (Grobe et al., 2007; Kassiri et al., 2009b; Gurley et al., 2006; Gurley \& Coffman, 2008). It is also important to consider age as an important factor affecting cardiac function of ACE2 KO. A study performed in our laboratories showed a cardiac diastolic dysfunction in 48 week old ACE2 KO mice (data not shown). Previous studies in ACE2 KO showed that infusion of Ang II caused concentric remodeling of the LV associated with mild diastolic dysfunction (Zhong et al., 2010). The experiments in the present study used a higher pressor dose of Ang II (1000 $\mathrm{ng} / \mathrm{kg} / \mathrm{min}$ for 4 weeks) to explore the peptide's effect on blood pressure and myocardial capacity in the ACE2 KO. The high dose of Ang II produced a maximal MAP increase of almost $42 \mathrm{mmHg}$ with similar changes in the WT and ACE2KO. The BP change was associated with a marked deficit in cardiac function in ACE2 KO. In addition to diastolic dysfunction, which was seen by the exacerbated E/A ratio, Ang II ACE2 KO exhibited severe systolic dysfunction. This was shown by the increase in the LVESd which resulted in a decrease in FS\% ( $30 \%)$ and a corresponding decrease in EF\% ( 32\%) in the ACE2 KO. Thus, the Echo data indicate that ACE2 plays protective role in conserving myocardial contractility function against long term Ang II stimulation.

Ang II infusion raised blood pressure to almost the same extent in both strains. Those results could be explained by two theories: 
1) Since plasma Ang II is the main factor responsible for the increased BP, the absence of a BP effect in ACE2 deletion corresponds to the fact that ACE2 was not detected in the plasma of normal mice (Elased, Cunha, Gurley, Coffman, \& Morris, 2006). Consistent with that, studies have shown cardiac administration of lentiviral vector coding for ACE2 (Diez-Freire et al., 2006) or treatment with recombinant human ACE2 (Zhong et al., 2010) effectively decreased systolic blood pressure. In contrast, neither long term infusion of Ang (1-7) (Grobe et al., 2007; McCollum, Gallagher, \& Ann, 2012) nor its overexpression (Mercure et al., 2008) could accentuate Ang II's pressor effects, underscoring a role for ACE2, but not plasma Ang (1-7), as a potential agent responsible for lowering BP.

2) The second theory is based on the fact that Ang II can be metabolized by enzymes other than ACE2, namely prolylcarboxypeptidase (PCP), prolylendopeptidase (PEP), and amino peptidase A (APA) (Grobe, Elased, Cool, \& Morris, 2012; Karamyan \& Speth, 2007). Special interest has been paid to APA (Ahmad \& Ward, 1990; Nomura et al., 2005), advocating that these enzymes might be functioning as a stockpile that preserves the body from the surplus of plasma Ang II and sustains the elevation in BP even in the absence of ACE2.

Whether it is just the increase in Ang II or the combination with the decrease in its metabolite, Ang (1-7), that plays the key role in mediating cardiac dysfunction and hypertrophy is still a point of debate. Support is greatest for the first theory (Crackower et al., 2002; Nakamura et al., 2008; Nako et al., 2012), however, there is still evidence that Ang (1-7) is essential for maintenance of normal cardiac function by counteracting Ang II induced hypertrophic effects (Grobe et al., 2007; Kassiri et al., 2009b). The results 
from the present study show a protective action of ACE2 against Ang II mediated cardiac hypertrophic cardiomyopathy. This was seen by the higher heart weight/ body weight ratio in Ang II ACE2 KO as compared to the Ang II WT. In addition, Echo data showed an increase in the posterior wall thickness in Ang II ACE2 KO compared to Ang II WT. Furthermore, the larger cardiomyocyte size measured within the LV of Ang II ACE2 KO mice clearly indicates a role of ACE2 in ameliorating Ang II mediating myocardiocyte hypertrophy. Results signify myocardial adaptive effects of ACE2 against hypertrophic response resulted from Ang II stimulation.

There are questions as to the mechanisms by which Ang II induces cardiac injury. One proposed mechanism is increased sarcolemmic and microvascular permeability leading to cardiac myocyte death (Gavras \& Gavras, 2002b). Ang II can also mediate cardiac injury through activation of JAK-STAT intracellular signaling which triggers inflammatory and remodeling responses (Dostal et al., 1997). Additionally, Ang II can mediate oxidative stress via activation of NADPH through AT1R activation and the subsequent formation of ROS (Browe \& Baumgarten, 2004) or via stimulation of myocardial collagen production and secretion (Grobe et al., 2007).

Ang II induced oxidative stress is likely one of the main mechanisms for peptide mediated cardiac damage, acting via AT1R overstimulation (Touyz, 2004; Byrne et al., 2003). Increased ROS can mediate myocardial hypertrophy and /or remodeling by varieties of mechanisms (Takimoto \& Kass, 2007; Tsutsui, Kinugawa, \& Matsushima, 2009; Hori \& Nishida, 2009). It has been confirmed that $\mathrm{O}_{2}^{-}$is the dominant ROS species in ACE2 KO heart (Oudit et al., 2007). Interestingly, Nako et al. reported that irbesartan (AT1R blocker) and Tempol (free radical scavenger) display the same 
cardioprotective effects against Ang II induced cardiac injury (Nako et al., 2012). Present study results show that there was enhanced $\mathrm{O}_{2}^{-}$production in the myocardium and aorta of ACE2 KO mice infused with Ang II. These results are consistent with a study by Oudit et al. (2007), which documented myocardial damage in ACE2 KO is mainly mediated by Ang II induced oxidative stress (Oudit et al., 2007). The degree of stimulation was especially evident in the aorta which showed high levels of DHE in the adventitia. The exact role of ACE2 in abrogating ROS formation is not fully understood. One theory is that the metabolism of Ang II to Ang (1-7) prevents Ang II induced oxidative stress. Another explanation is that ACE2 acts as a scavenger against ROS formation. Collectively, these findings emphasize the importance of oxidative stress in Ang II induced cardiac and vascular injury.

Another crucial aspect of Ang II induced cardiovascular pathologies is that Ang II stimulation can induce myocardial fibrosis and remodeling (Letavernier et al., 2008; Burlew \& Weber, 2002). Chronic Ang II treatment stimulates a maladaptive response in cardiomyocytes which leads to differential gene expressions, production of ROS, impaired calcium cycling (Egger \& Domenighetti, 2010), and stimulation of myocardial and aortic collagen deposition which are signs of cardiovascular remodeling (Letavernier et al., 2008). Using two different types of collagen stains, present results show elevated myocardium collagen content in Ang II ACE2 KO. Consistent with that, Zhong et al. (2010) reported that Ang II infusion resulted in greater expression of procollagen type Ia1. It is plausible that the high collagen and resultant fibrosis seen in ACE2 KO mice might result in cardiac stiffness and decreased compliance which are markers for diastolic dysfunction (Moreo et al., 2009; Burlew \& Weber, 2002). Moreover, the higher E/A ratio 
as well as the increased relaxation time seen in Ang II ACE2 KO verify the diastolic dysfunction. The structural changes are likely responsible for diminished function, that is, the decrease in FS\% (more than 30\%), and EF\% ( 31\%). Interestingly, results from collagen staining in the aorta also suggest a protective effect of ACE2 in this large transport vessel. In fact, this is the first report to suggest that the aorta is an important part of the Ang II/ACE2 pathological cascade. Vascular fibrosis involves media accumulation of extracellular matrix proteins, such as collagen, elastin, fibronectin and proteoglycans. Aortas from Ang II ACE2 KO show higher collagen in the adventitia layer along with a reduced luminal size area which suggests decreased compliance. This would result in aorta stiffness which can negatively affect cardiac afterload and further worsen $\mathrm{EF} \%$. There is no information on changes in the smaller resistance vessels in ACE2 KO. However, since BP is not different between groups, one might suspect that the vascular fibrotic changes would be similar. Ang II induced vascular smooth muscle growth, arterial remodeling and inflammation associated with cellular growth and calcification is well documented (Cheng et al., 2011; Touyz, 2005; Touyz, 2004; Savoia, Burger, Nishigaki, Montezano, \& Touyz, 2011). It is reasonable that the presence of ACE2 in the vascular endothelial cell and arterial smooth muscle cells (Donoghue et al., 2000; Hamming et al., 2004) could function protectively in those vessels from the hypertrophic effect of Ang II (Hayashi et al., 2010; Jin, Lu, \& Lin, 2011). Moreover, it has been shown that ACE2 upregulation in the aorta in response to AT1 receptor blocker increased the lumen of the aorta in SHR (Igase, Strawn, Gallagher, Geary, \& Ferrario, 2005). These data suggest specific protective role of ACE2 to the maladaptive cardiovascular remodeling in response to Ang II stimulation. 


\section{CONCLUSION}

The RAS system is the driving force that governs the progression and complication of CVD and the associated target organ damage. ACE2's main role is to shuttle the vasopressor Ang II and tip the balance toward Ang (1-7) formation. Ang (1-7) has beneficial effects since it counteracts Ang II mediated effects. So, ACE2 plays a particular protective role as a negative regulator of the RAS. The hypothesis proposed in this study was that deletion of ACE2 gene results in enhanced Ang II induce myocardium and aortic remodeling.

Chronic Ang II infusion (1000 ng/kg/min for 4weeks) caused similar elevation in MAP in the WT and ACE2 KO mice. However, the elevated MAP was associated with cardiac systolic and diastolic dysfunction in ACE2 KO mice but not in the WT group. Cardiac dysfunction was shown by the decreased $\mathrm{FS} \%$ and $\mathrm{EF} \%$ as well as the lowered E/A ratio in Ang II infused ACE2 KO compared to WT. Moreover, results from cardiomyocytes size analysis showed an increase in the cardiomyocyte cross sectional area in ACE2 KO which is an indication of hypertrophic cardiomyopathy. Consistent with that, heart weight / body weight analysis showed higher ratio in Ang II infused ACE2 KO mice compared to WT. Collagen staining results showed an overt higher collagen content within the myocardium of Ang II ACE2 KO which indicates a myocardial remodeling. Moreover, higher collagen content observed within the adventitial layer of the ascending thoracic aorta in ACE2 KO which suggests an aortic remodeling. Furthermore, higher ROS formation was observed within the myocardium and aorta of Ang II infused ACE2 KO group. In summary, findings support the hypothesis that ACE2 is a crucial enzyme that modulates Ang II induce myocardial maladaptive hypertrophic response and 
cardiovascular remodeling in mice. This study presents explanations for the protective effects of ACE2 against the deleterious effects of Ang II and its cardiac sequelae. The work herein magnifies the role of ACE2 as a potential candidate targeting cardiovascular disease progression and supports the hypothesis that ACE2 might be a promising avenue for developing cardiovascular therapeutic agents. 


\title{
APPENDIX A
}

\section{Echocardiography shows impaired cardiac response to Ang II in ACE2 KO mice}

Mahmoud Alghamri, Nathan Weir, Joshua Miller, Zhongyu Yan, Mark Anstadt,

\author{
Mariana Morris
}

Department of Pharmacology and Toxicology, Boonshoft School of Medicine, Wright

State University, Dayton, $\mathrm{OH}$

Angiotensin converting enzyme 2 (ACE2) is a monocarboxypeptidase that metabolizes the potent vasoconstrictor, angiotensin II (Ang II), to the vasodilator, Ang (1-7). Since ACE2 is thought to regulate cardiac function, we hypothesized that loss of ACE2 would negatively affect cardiac function and alter the response to Ang II. To assess cardiac function, echocardiographic (Echo) measurements were made in control and ACE2 knockout (ACE2 KO) mice under baseline and Ang II stimulated conditions (1000 $\mathrm{ng} / \mathrm{kg} / \mathrm{min}$ Ang II for 4 weeks). Echos were made using a Siemens® ACUSON system. Left ventricular end diastolic and end systolic areas as well as dimensions (LVEDa, LVESa, LVEDd and LVESd) were measured during 2-D and M-Modes. Fractional shortening (FS) was calculated from M-Mode measurements of left ventricular dimensions. Posterior wall thickness (PWT) and septal wall thickness (SWT) were measured in M-Mode. Baseline Echos showed higher LVESd in ACE2 KO compared to control ( 0.25 vs $0.22 \mathrm{~cm} ; \mathrm{p}<0.05$, respectively). This corresponds to a decrease in FS in ACE2 KO (0.40 vs 0.35; $\mathrm{p}<0.05)$. There was also an increase in the LVESa in ACE2 KO compared to control ( 0.15 vs $0.12 \mathrm{~cm} 2 ; \mathrm{p}<0.05)$. Chronic Ang II infusion produced an initial increase in the mean blood pressure in both groups in the first week (96 to 135 $\mathrm{mmHg}$ in ACE2 KO vs 103 to $145 \mathrm{mmHg}$ in control). This was associated with an increase in LVESd in ACE2 KO ( +13\%; 0.25 vs $0.28 \mathrm{~cm}$; $\mathrm{p}<0.001)$ and controls $(\sim+11 \% ; 0.22$ vs $0.25 \mathrm{~cm} ; \mathrm{p}<0.05)$. These changes led to a significant decrease in FS in ACE2 KO in response to Ang II $(\sim-20 \%$; 0.35 vs 0.28 ; $p<0.05)$ with no change in controls (0.40 vs 0.35 ; NS). Moreover, LVESa was increased with Ang II treatment in ACE2 KO ( +19\%; 0.14 vs $0.17 \mathrm{~cm} 2 ; \mathrm{p}<0.01)$ and controls $(\sim+16 \% ; 0.13$ vs $0.15 \mathrm{~cm} 2$; $\mathrm{p}<0.01)$. There were no changes in LVEDa or LVEDd and no signs of dilated wall thickness as shown by PWT and SWT. Results suggest a specific cardioprotective role for ACE2 at the baseline and after long term Ang II stimulated conditions.

\footnotetext{
Abstract was published in Hypertension (Hypertension. 2011;58:e33-e183). Abstract was presented in HBPR2011, Orlando, FL.
} 


\section{APPENDIX B}

\section{Effect of Chronic Swim Exercise on Adiposity and Metabolic Function in Mice}

Roberta L. Pohlman, Mahmoud S. Alghamari, Ana Carneiro, Lynn Hartzler, L. David Mirkin, Vera Farah ,Mariana Morris

Department of Pharmacology and Toxicology, Boonshoft School of Medicine, Wright State University, Dayton, OH

A western diet, high in fat and sugar, causes metabolic and cardiovascular dysfunction. The objective was to determine the effect of moderate swimming exercise $(1 \mathrm{hr} / \mathrm{d}, 3$ $\mathrm{d} / \mathrm{wk}, \mathrm{n}=9 / 10$ per group) on the metabolic and cardiac responses to a high fat/sugar diet in mice. Groups are: Control (CON); Sedentary (SED, fat/fructose) and Exercise (EX, fat/fructose). Chow (60\% fat) and fructose water (10\%) were given ad libitum for $8 \mathrm{wk}$. Parameters measured were body fat, heart function (echocardiography, Echo), glucose, glucose tolerance (GTT) and liver glycogen. Mice gained body fat (up to $45 \%$ ) on the fat/fructose diet with no difference between groups. Metabolic function was improved in the EX group as determined by glucose (SED>EX, $\mathrm{p}<0.01)$ and GTT (SED>EX, $\mathrm{p}<0.05)$ Insulin was lower in EX than SED, suggesting improvement in function. The diet increased liver glycogen, an effect which was improved in the EX group. Echo measurements showed impairment in cardiac function in the EX group. Relative wall thickness was greater in EX vs. SED $(0.35 \square 0.01$ vs. $0.29 \square 0.01, \mathrm{p}<0.01)$. Fractional shortening was reduced in EX group (EX $0.31 \square 0.01$ vs. SED 0.36 $\square 0.01, \mathrm{p}<0.01$ ). This resulted in a reduced ejection fraction of $\sim 37 \%$ (EX) vs. $49 \%$ (SED). Data demonstrate that a moderate exercise paradigm combined with a high fat/sugar diet improves metabolic function, but is detrimental to heart function in morbidly obese mice.

Poster presented at the Experimental Biology (EB) meeting 2012; San Diego, CA. 


\begin{abstract}
APPENDIX C
Echocardiography shows impaired cardiac function in ACE2 knockout mice

\author{
Alghamri M, Weir N, Miller J, Anstadt M, Morris M \\ Department of Pharmacology and Toxicology, Boonshoft School of Medicine, Wright \\ State University, Dayton, $\mathrm{OH}$
}

Angiotensin converting enzyme2 (ACE2) is a monocarboxypeptidase that metabolizes the potent vasoconstrictor peptide, angiotensin II (Ang II), to the vasodilator Ang (1-7). Since ACE2 is thought to regulate heart function, we hypothesized that loss of ACE2 would negatively affect function and alter the response to Ang II. Echocardiographic (Echo) measurements were made in control and ACE2 knockout (KO) mice under baseline and Ang II stimulated conditions (1000 ng Ang II/ $/ \mathrm{kg} / \mathrm{min}$ for 4 weeks). Echos were recorded using a Siemens ${ }^{\circledR}$ ACUSON system. Images of the left ventricle were taken in the M-Mode and 2-D Mode. left ventricular end diastolic and end systolic areas as well as dimensions (LVEDa, LVESa, LVEDd, and LVESd) respectively, were measured. Ejection fraction (EF) was calculated. Posterior wall thickness (PWT) and septal wall thickness (SWT) were measured in the M-Mode. Baseline Echo measurements show higher lVESa $(\sim+10 \%)$ in ACE2KO vs Control. This corresponds to a decrease in $\mathrm{EF}(\sim-25 \%)$. Ang II infusion produced a marked increase in lVESa $(\sim+19 \%)$ in ACE2KO mice as compared to controls $(\sim+9 \%)$. This meant that there was a greater decrease in EF in KO mice $(\sim-165 \%)$ vs controls $(\sim-125 \%)$. There was no sign of dilated cardiomyopathy as shown by PWT and SWT measurements. Results suggest a protective role for ACE2 in the control of cardiac contractile function under baseline and Ang II stimulation.

Poster presented at Celebration of Research, Scholarship, and Creative Activities 2011, Wright State University, Dayton, $\mathrm{OH}$. 


\section{APPENDIX D}

\section{Enhanced Angiotensin II-Induced Cardiac and Aortic Remodeling in ACE2 Knockout Mice}

Mahmoud Alghamri, Nathan Weir, Mark Anstadt, Khalid Elased, Mariana Morris

Department of Pharmacology and Toxicology, Boonshoft School of Medicine, Wright State University, Dayton, $\mathrm{OH}$

Activation of the renin angiotensin system (RAS) is a well-known driving force, governing the aggravation and progression of CVD and associated target organ damage. Angiotensin (Ang) converting enzyme 2 (ACE2), a new member within the RAS family, was first cloned from the ventricle of patient with heart failure. ACE2 was shown to be upregulated under pathological conditions. We hypothesize that loss of ACE2 negatively affects cardiac function and exacerbates hypertrophic cardiomyopathy and aortic remodeling in response to Ang II. 8 weeks old ACE2 knock out (KO) and wild type (WT) mice were infused with Ang II s.c. (1000 $\mathrm{ng} / \mathrm{kg} / \mathrm{min}$ for 4 weeks) using osmotic pumps. Blood pressure (radiotelemetry), cardiac function (echocardiography) and cardiac/aortic structural damage (histology) were examined. At baseline before Ang II, ACE2 KO displayed a normal phenotype for cardiac function and blood pressure. After 4 weeks of Ang II infusion, the mean arterial pressure (MAP) was increased ( 40\% in WT and $\sim 33 \%$ in ACE2 KO) with no differences between groups. However, ACE2 KO responded differently to the increased MAP. Cardiac function analysis revealed severe myocardial dysfunction shown by the lowered EF\% (31\% vs 13\%) as well as FS\% (30\% vs 6\%) in ACE2 KO vs WT, respectively. In addition, the cardiac dysfunction was associated with hypertrophic cardiomyopathy shown by the increased left ventricular wall thickness as well as heart weight/ body weight ratio. Moreover, collagen staining in the myocardium and aorta revealed higher collagen percentage in ACE2 KO which indicates cardiovascular remodeling. Furthermore, results showed enhanced oxidative stress in the myocardium and aorta of Ang II infused ACE2 KO compared to Ang II WT. In conclusion, ACE2 attenuates myocardial maladaptive hypertrophy and cardiovascular remodeling in response to long term Ang II stimulation in mice.

Poster presented during Indiana- Ohio Discussion Group (IODG) 2011, Indianapolis, ID. 


\section{APPENDIX E}

\section{Role of angiotensin converting enzyme 2 (ACE2) in body fat and glucose tolerance}

Nathan Weir, Mahmoud Alghamri, Danielle Barnhart, Zhongyu Yan, Khalid Elased, Mariana Morris

Department of Pharmacology and Toxicology, Boonshoft School of Medicine, Wright State University, Dayton, $\mathrm{OH}$

Studies in diabetic humans have found dysregulation of the renin angiotensin system (RAS), specifically angiotensin (Ang) II. Angiotensin converting enzyme (ACE) 2 is often upregulated as a compensatory enzyme during times of cardiovascular and metabolic stress. We hypothesize global loss of ACE2 will negatively affect glucose homeostasis in mice with diet induced obesity. To study this hypothesis, ACE2 knockout (KO) and wild type (WT) mice were fed either a 60\% high fat (HF) chow for 12 weeks or maintained on normal chow (NC). At 8 weeks of age before initiation of the diet, basic metabolic parameters were measured using QNMR for body fat analysis. Both strains had similar baseline body weight ( $\mathrm{WT}=23.90 \pm 0.76, \mathrm{KO}=23.70 \pm 0.75 \mathrm{~g}$ ) but $\mathrm{KO}$ mice showed increased percent body fat [WT=4.51 $\pm 0.80 \%(1.08$ of $23.91 \mathrm{~g}), \mathrm{KO}=7.01 \pm 0.76 \%$ (1.66 of $23.69 \mathrm{~g})$ ] and increased fed glucose $(\mathrm{WT}=147.9 \pm 4.53, \mathrm{KO}=164.1 \pm 5.31 \mathrm{mg} / \mathrm{dl})$. Daily caloric intake was comparable between strains (WT $=11.84 \pm 0.37, \mathrm{KO}=11.49 \pm 1.27 \mathrm{kcal}$ ). After 12 weeks of diet, both WT and KO mice had gained significant body weight from baseline (WT 13.96, $\mathrm{KO}=14.63 \mathrm{~g}$ ) and differences in body fat were eliminated, however, glucose handling was altered. Fed glucose showed a main effect for both strain $(\mathrm{WT}=140.31 \pm 4.00, \mathrm{KO}=156.83 \pm 4.23 \mathrm{mg} / \mathrm{dl})$ and $\operatorname{diet}(\mathrm{NC}=141.67 \pm 3.78$, $\mathrm{HF}=155.48 \pm 4.47 \mathrm{mg} / \mathrm{dl})$. Fasted glucose revealed a strain and diet interaction $(\mathrm{NCxWT}=101.11 \pm 5.00, \mathrm{NCxKO}=94.44 \pm 5.00, \mathrm{HFxWT}=105.83 \pm 6.13$, $\mathrm{HFxKO}=138.14 \pm 5.67 \mathrm{mg} / \mathrm{dl})$. Additionally, an intraperitoneal glucose tolerance test showed HF KO mice had a significantly elevated area under the curve compared to HF WT mice (WT=311.1 $\pm 20.20, \mathrm{KO}=382 \pm 18.26 \mathrm{mg} / \mathrm{dl} / \mathrm{min}$ ) while $\mathrm{NC}$ WT and NC $\mathrm{KO}$ had overlapping curves $(\mathrm{WT}=237.0 \pm 9.12, \mathrm{KO}=239.0 \pm 7.92 \mathrm{mg} / \mathrm{dl} / \mathrm{min})$. Plasma analysis revealed decreased circulating insulin in $\mathrm{NC} \mathrm{KO}$ mice $(\mathrm{WT}=1.1 \pm 0.075, \mathrm{KO}=0.86 \pm 0.056$ $\mathrm{ng} / \mathrm{ml}$ ). High fat diet increased insulin in both strains, but more so in WT (HF $\mathrm{WT}=3.8 \pm 0.63$, HF KO=3.0 $\pm 0.61 \mathrm{ng} / \mathrm{ml})$. HF WT mice also demonstrated increased pancreas ACE2 activity (NC WT $=0.56 \pm 0.13$, HF WT $=2.14 \pm 0.5 \mathrm{pmol} \mathrm{MCA} / \mu \mathrm{g} / \mathrm{h}$ ). These combined results indicate loss of ACE2 negatively affects glucose homeostasis possibly through insulin handling in the pancreas.

Poster presented in Ohio Physiological Society (OPS), 2011, University of Cincinnati, Cincinnati, $\boldsymbol{O H}$. 


\section{Reference List}

Ahmad, S. \& Ward, P. E. (1990). Role of aminopeptidase activity in the regulation of the pressor activity of circulating angiotensins. J.Pharmacol.Exp.Ther., 252, 643-650.

Benov, L., Sztejnberg, L., \& Fridovich, I. (1998). Critical evaluation of the use of hydroethidine as a measure of superoxide anion radical. Free Radic.Biol.Med., 25, 826831.

Braun-Menendez, E. \& PAGE, I. H. (1958). Suggested Revision of Nomenclature--Angiotensin. Science, 127, 242.

Browe, D. M. \& Baumgarten, C. M. (2004). Angiotensin II (AT1) receptors and NADPH oxidase regulate $\mathrm{Cl}$ - current elicited by betal integrin stretch in rabbit ventricular myocytes. J.Gen.Physiol, 124, 273-287.

Burlew, B. S. \& Weber, K. T. (2002). Cardiac fibrosis as a cause of diastolic dysfunction. Herz, 27, 92-98.

Burrell, L. M., Johnston, C. I., Tikellis, C., \& Cooper, M. E. (2004). ACE2, a new regulator of the renin-angiotensin system. Trends Endocrinol.Metab, 15, 166-169.

Byrne, J. A., Grieve, D. J., Bendall, J. K., Li, J. M., Gove, C., Lambeth, J. D. et al. (2003). Contrasting roles of NADPH oxidase isoforms in pressure-overload versus angiotensin II-induced cardiac hypertrophy. Circulation Research, 93, 802-805. 
Chappell, M. C., Allred, A. J., \& Ferrario, C. M. (2001). Pathways of angiotensin(1-7) metabolism in the kidney. Nephrol.Dial.Transplant., 16 Suppl 1, 22-26.

Cheng, J. F., Ni, G. H., Chen, M. F., Li, Y. J., Wang, Y. J., Wang, C. L. et al. (2011). Involvement of profilin-1 in angiotensin II-induced vascular smooth muscle cell proliferation. Vascul.Pharmacol., 55, 34-41.

Collins, K. A., Korcarz, C. E., \& Lang, R. M. (2003). Use of echocardiography for the phenotypic assessment of genetically altered mice. Physiol Genomics, 13, 227239.

Crackower, M. A., Sarao, R., Oudit, G. Y., Yagil, C., Kozieradzki, I., Scanga, S. E. et al. (2002). Angiotensin-converting enzyme 2 is an essential regulator of heart function. Nature, 417, 822-828.

De Mello, W. C. \& Specht, P. (2006). Chronic blockade of angiotensin II AT1receptors increased cell-to-cell communication, reduced fibrosis and improved impulse propagation in the failing heart. J.Renin.Angiotensin.Aldosterone.Syst., 7, 201-205.

de Gasparo, G. M. (2002). [AT(1) and AT(2) angiotensin II receptors: key features]. Drugs, 62 (S8), 1-10.

de Gasparo , G. M., Catt, K. J., Inagami, T., Wright, J. W., \& Unger, T. (2000). International union of pharmacology. XXIII. The angiotensin II receptors. Pharmacol.Rev., 52 (3), 415-472. 
Diez-Freire, C., Vazquez, J., Correa de Adjounian, M. F., Ferrari, M. F., Yuan, L., Silver, X. et al. (2006). ACE2 gene transfer attenuates hypertension-linked pathophysiological changes in the SHR. Physiol Genomics, 27, 12-19.

Donoghue, M., Hsieh, F., Baronas, E., Godbout, K., Gosselin, M., Stagliano, N. et al. (2000). A novel angiotensin-converting enzyme-related carboxypeptidase (ACE2) converts angiotensin I to angiotensin 1-9. Circulation Research, 87, E1-E9.

Dostal, D. E., Hunt, R. A., Kule, C. E., Bhat, G. J., Karoor, V., McWhinney, C. D. et al. (1997). Molecular mechanisms of angiotensin II in modulating cardiac function: intracardiac effects and signal transduction pathways. J.Mol.Cell Cardiol., 29, 28932902.

Egger, M. \& Domenighetti, A. A. (2010). Adaptive and maladaptive remodeling of cardiomyocyte excitation-contraction coupling by angiotensin II. Trends Cardiovasc.Med., 20, 78-85.

Elased, K. M., Cunha, T. S., Gurley, S. B., Coffman, T. M., \& Morris, M. (2006). New mass spectrometric assay for angiotensin-converting enzyme 2 activity. Hypertension, 47, 1010-1017.

Elased, K. M., Cunha, T. S., Coffman, T. M., Gurley, S. B., \& Morris, M. (2005). A novel assay for measurement of angiotensin converting enzyme 2 (ACE2) activity. Circulation, 112, U204.

Emilsson, K., Egerlid, R., Nygren, B. M., \& Wandt, B. (2006). Mitral annulus motion versus long-axis fractional shortening. Exp.Clin.Cardiol., 11, 302-304. 
Fasciolo, J. C., Houssay, B. A., \& Taquini, A. C. (1938). The blood-pressure raising secretion of the ischaemic kidney. J.Physiol, 94, 281-293.

Ferrario, C. M., Chappell, M. C., Tallant, E. A., Brosnihan, B. K., \& Diz, D. I. (1997). Counterregulatory actions of angiotensin-(1-7). Hypertension 30[Pt 2], 535-541.

Ferreira, A. J., Santos, R. A., Bradford, C. N., Mecca, A. P., Sumners, C., Katovich, M. J. et al. (2010). Therapeutic implications of the vasoprotective axis of the renin-angiotensin system in cardiovascular diseases. Hypertension, 55, 207-213.

Gao, X. M., Dart, A. M., Dewar, E., Jennings, G., \& Du, X. J. (2000). Serial echocardiographic assessment of left ventricular dimensions and function after myocardial infarction in mice. Cardiovasc.Res., 45, 330-338.

Gardin, J. M., Siri, F. M., Kitsis, R. N., Edwards, J. G., \& Leinwand, L. A. (1995). Echocardiographic assessment of left ventricular mass and systolic function in mice. Circulation Research, 76, 907-914.

Gavras, I. \& Gavras, H. (2002a). Angiotensin II as a cardiovascular risk factor. J.Hum.Hypertens., 16 Suppl 2, S2-S6.

Gavras, I. \& Gavras, H. (2002b). Angiotensin II as a cardiovascular risk factor. J.Hum.Hypertens., 16 Suppl 2, S2-S6.

Goldblatt, H., Lynch, J., Hanzal, R. F., \& Summerville, W. W. (1934). Studies on experimental hypertension : I. The production of persistent elevation of systolic blood pressure by means of renal ischemia. J.Exp.Med., 59, 347-379. 
Grobe, J. L., Mecca, A. P., Lingis, M., Shenoy, V., Bolton, T. A., Machado, J. M. et al. (2007). Prevention of angiotensin II-induced cardiac remodeling by angiotensin-(17). Am.J.Physiol. Heart Circ.Physiol., 292, H736-H742.

Grobe, N., Elased, K. M., Cool, D. R., \& Morris, M. (2012). Mass spectrometry for the molecular imaging of angiotensin metabolism in kidney. Am.J.Physiol Endocrinol.Metab, 302, E1016-E1024.

Gurley, S. B., Allred, A., Le, T. H., Griffiths, R., Mao, L., Philip, N. et al. (2006). Altered blood pressure responses and normal cardiac phenotype in ACE2-null mice. J.Clin.Invest, 116 (8), 2218-2225.

Gurley, S. B. \& Coffman, T. M. (2008). Angiotensin-converting enzyme 2 gene targeting studies in mice: mixed messages. Exp.Physiol, 93, 538-542.

Hamming, I., Timens, W., Bulthuis, M. L., Lely, A. T., Navis, G. J., \& van, G. H. (2004). Tissue distribution of ACE2 protein, the functional receptor for SARS coronavirus. A first step in understanding SARS pathogenesis 3. J.Pathol., 203, 631-637.

Harmer, D., Gilbert, M., Borman, R., \& Clark, K. L. (2002). Quantitative mRNA expression profiling of ACE 2, a novel homologue of angiotensin converting enzyme. FEBS Lett., 532, 107-110.

Hartley, C. J., Taffet, G. E., Reddy, A. K., Entman, M. L., \& Michael, L. H. (2002). Noninvasive cardiovascular phenotyping in mice. ILAR.J., 43, 147-158. 
Hayashi, N., Yamamoto, K., Ohishi, M., Tatara, Y., Takeya, Y., Shiota, A. et al. (2010). The counterregulating role of ACE2 and ACE2-mediated angiotensin 1-7 signaling against angiotensin II stimulation in vascular cells. Hypertens.Res., 33, 11821185.

Hori, M. \& Nishida, K. (2009). Oxidative stress and left ventricular remodelling after myocardial infarction. Cardiovasc.Res., 81, 457-464.

Igase, M., Strawn, W. B., Gallagher, P. E., Geary, R. L., \& Ferrario, C. M. (2005). Angiotensin II AT1 receptors regulate ACE2 and angiotensin-(1-7) expression in the aorta of spontaneously hypertensive rats. Am.J.Physiol Heart Circ.Physiol, 289, H1013H1019.

Jin, X. Q., Lu, Z. Q., \& Lin, X. (2011). [Effect of ACE2 gene transfection on the proliferation of vascular smooth muscle cells in rats]. Zhonghua Yi.Xue.Za Zhi., 91, 125128.

Karamyan, V. T. \& Speth, R. C. (2007). Enzymatic pathways of the brain reninangiotensin system: unsolved problems and continuing challenges. Regul.Pept., 143, 1527.

Kassiri, Z., Zhong, J., Guo, D., Basu, R., Wang, X., Liu, P. P. et al. (2009a). Loss of angiotensin-converting enzyme 2 accelerates maladaptive left ventricular remodeling in response to myocardial infarction. Circ.Heart Fail., 2, 446-455. 
Kassiri, Z., Zhong, J., Guo, D., Basu, R., Wang, X., Liu, P. P. et al. (2009b). Loss of angiotensin-converting enzyme 2 accelerates maladaptive left ventricular remodeling in response to myocardial infarction. Circ.Heart Fail., 2, 446-455.

Kirchhoff, F., Krebs, C., Abdulhag, U. N., Meyer-Schwesinger, C., Maas, R., Helmchen, U. et al. (2008). Rapid development of severe end-organ damage in C57BL/6 mice by combining DOCA salt and angiotensin II. Kidney International, 73, 643-650.

Kuecherer, H. F., Kee, L. L., Modin, G., Cheitlin, M. D., \& Schiller, N. B. (1991). Echocardiography in serial evaluation of left ventricular systolic and diastolic function: importance of image acquisition, quantitation, and physiologic variability in clinical and investigational applications. J.Am.Soc.Echocardiogr., 4, 203-214.

Kunikullaya, U. K., Ananthakrishnan, V., \& Goturu, J. (2011). Robert Tigerstedt and the discovery of renin - A revisit. Int.J.Cardiol..

Letavernier, E., Perez, J., Bellocq, A., Mesnard, L., de Castro, K. A., Haymann, J. P. et al. (2008). Targeting the calpain/calpastatin system as a new strategy to prevent cardiovascular remodeling in angiotensin II-induced hypertension. Circulation Research, 102, 720-728.

Liu, J. \& Rigel, D. F. (2009). Echocardiographic examination in rats and mice. Methods Mol.Biol., 573, 139-155.

Mazzolai, L., Nussberger, J., Aubert, J. F., Brunner, D. B., Gabbiani, G., Brunner, H. R. et al. (1998). Blood pressure-independent cardiac hypertrophy induced by locally activated renin-angiotensin system. Hypertension, 31, 1324-1330. 
McCollum, L. T., Gallagher, P. E., \& Ann, T. E. (2012). Angiotensin-(1-7) attenuates angiotensin II-induced cardiac remodeling associated with upregulation of dual-specificity phosphatase 1. Am.J.Physiol Heart Circ.Physiol, 302, H801-H810.

Melaragno, M. G. \& Fink, G. D. (1996). Slow pressor effect of angiotensin II in normotensive rats with renal artery stenosis. Clin.Exp.Pharmacol.Physiol, 23, 140-144.

Mercure, C., Yogi, A., Callera, G. E., Aranha, A. B., Bader, M., Ferreira, A. J. et al. (2008). Angiotensin(1-7) blunts hypertensive cardiac remodeling by a direct effect on the heart. Circulation Research, 103, 1319-1326.

Moreo, A., Ambrosio, G., De, C. B., Pu, M., Tran, T., Mauri, F. et al. (2009). Influence of myocardial fibrosis on left ventricular diastolic function: noninvasive assessment by cardiac magnetic resonance and echo. Circ.Cardiovasc.Imaging, 2, 437443.

Nakamura, K., Koibuchi, N., Nishimatsu, H., Higashikuni, Y., Hirata, Y., Kugiyama, K. et al. (2008). Candesartan ameliorates cardiac dysfunction observed in angiotensin-converting enzyme 2-deficient mice. Hypertens.Res., 31, 1953-1961.

Nako, H., Kataoka, K., Koibuchi, N., Dong, Y. F., Toyama, K., Yamamoto, E. et al. (2012). Novel mechanism of angiotensin II-induced cardiac injury in hypertensive rats: the critical role of ASK1 and VEGF. Hypertens.Res., 35, 194-200.

Ng, K. K. \& Vane, J. R. (1967). Conversion of angiotensin I to angiotensin II. Nature, 216, 762-766. 
Nomura, M., Nomura, S., Mitsui, T., Suzuki, M., Kobayashi, H., Ito, T. et al. (2005). Possible involvement of aminopeptidase A in hypertension and renal damage in Dahl salt-sensitive rats. American Journal of Hypertension, 18, 538-543.

Ohtsuki, M., Morimoto, S., Izawa, H., Ismail, T. F., Ishibashi-Ueda, H., Kato, Y. et al. (2010). Angiotensin converting enzyme 2 gene expression increased compensatory for left ventricular remodeling in patients with end-stage heart failure. Int.J.Cardiol., 145, $333-334$.

Oudit, G. Y., Kassiri, Z., Patel, M. P., Chappell, M., Butany, J., Backx, P. H. et al. (2007). Angiotensin II-mediated oxidative stress and inflammation mediate the agedependent cardiomyopathy in ACE2 null mice. Cardiovasc.Res., 75, 29-39.

Pacher, P., Nagayama, T., Mukhopadhyay, P., Batkai, S., \& Kass, D. A. (2008). Measurement of cardiac function using pressure-volume conductance catheter technique in mice and rats. Nat.Protoc., 3, 1422-1434.

PAGE, I. H. (1939). ON THE NATURE OF THE PRESSOR ACTION OF RENIN. J.Exp.Med., 70, 521-542.

Patten, R. D., Aronovitz, M. J., Bridgman, P., \& Pandian, N. G. (2002). Use of pulse wave and color flow Doppler echocardiography in mouse models of human disease. J.Am.Soc.Echocardiogr., 15, 708-714.

Peshavariya, H. M., Dusting, G. J., \& Selemidis, S. (2007). Analysis of dihydroethidium fluorescence for the detection of intracellular and extracellular superoxide produced by NADPH oxidase. Free Radic.Res., 41, 699-712. 
Picard, M. H., Popp, R. L., \& Weyman, A. E. (2008). Assessment of left ventricular function by echocardiography: a technique in evolution. J.Am.Soc.Echocardiogr., 21, 14-21.

Respress, J. L. \& Wehrens, X. H. (2010). Transthoracic echocardiography in mice. J.Vis.Exp.

Ruiz-Ortega, M., Lorenzo, O., Ruperez, M., Esteban, V., Mezzano, S., \& Egido, J. (2001a). Renin-angiotensin system and renal damage: emerging data on angiotensin II as a proinflammatory mediator. Contrib.Nephrol., 123-137.

Ruiz-Ortega, M., Lorenzo, O., Ruperez, M., Esteban, V., Suzuki, Y., Mezzano, S. et al. (2001b). Role of the renin-angiotensin system in vascular diseases: expanding the field. Hypertension, 38, 1382-1387.

Savoia, C., Burger, D., Nishigaki, N., Montezano, A., \& Touyz, R. M. (2011). Angiotensin II and the vascular phenotype in hypertension. Expert.Rev.Mol.Med., 13, e11.

Schiller, N. B. \& Foster, E. (1996). Analysis of left ventricular systolic function. Heart, 75, 17-26.

Skeggs, L. T., Jr., Kahn, J. R., \& Shumway, N. P. (1956). The preparation and function of the hypertensin-converting enzyme. J.Exp.Med., 103, 295-299.

Sohn, D. W., Kim, Y. J., Kim, H. C., Chun, H. G., Park, Y. B., \& Choi, Y. S. (1999). Evaluation of left ventricular diastolic function when mitral $\mathrm{E}$ and A waves are 
completely fused: role of assessing mitral annulus velocity. J.Am.Soc.Echocardiogr., 12, 203-208.

Takimoto, E. \& Kass, D. A. (2007). Role of oxidative stress in cardiac hypertrophy and remodeling. Hypertension, 49, 241-248.

Tanaka, N. (2003). [Non-invasive assessment of cardiac function]. Rinsho Byori, $51,751-760$.

Tanaka, N., Dalton, N., Mao, L., Rockman, H. A., Peterson, K. L., Gottshall, K. R. et al. (1996). Transthoracic echocardiography in models of cardiac disease in the mouse. Circulation, 94, 1109-1117.

Tipnis, S. R., Hooper, N. M., Hyde, R., Karran, E., Christie, G., \& Turner, A. J. (2000). A human homolog of angiotensin-converting enzyme. Cloning and functional expression as a captopril-insensitive carboxypeptidase. J Biol.Chem., 275, 33238-33243.

Touyz, R. M. (2004). Reactive oxygen species and angiotensin II signaling in vascular cells -- implications in cardiovascular disease. Braz.J.Med.Biol.Res., 37, 12631273.

Touyz, R. M. (2005). Intracellular mechanisms involved in vascular remodelling of resistance arteries in hypertension: role of angiotensin II. Exp.Physiol, 90, 449-455.

Tsutsui, H., Kinugawa, S., \& Matsushima, S. (2009). Mitochondrial oxidative stress and dysfunction in myocardial remodelling. Cardiovasc.Res., 81, 449-456. 
Vickers, C., Hales, P., Kaushik, V., Dick, L., Gavin, J., Tang, J. et al. (2002). Hydrolysis of biological peptides by human angiotensin-converting enzyme-related carboxypeptidase. J.Biol.Chem., 277, 14838-14843.

Vlahovic, A. \& Popovic, A. D. (1999). [Evaluation of left ventricular diastolic function using Doppler echocardiography]. Med.Pregl., 52, 13-18.

Wang, H. X., Zhang, Q. F., Zeng, X. J., Wang, W., Tang, C. S., \& Zhang, L. K. (2010). Effects of angiotensin III on protein, DNA, and collagen synthesis of neonatal cardiomyocytes and cardiac fibroblasts in vitro. J.Cardiovasc.Pharmacol.Ther., 15, 393402.

Xia, Y. \& Karmazyn, M. (2004). Obligatory role for endogenous endothelin in mediating the hypertrophic effects of phenylephrine and angiotensin II in neonatal rat ventricular myocytes: evidence for two distinct mechanisms for endothelin regulation. J.Pharmacol.Exp.Ther., 310, 43-51.

Xu, P., Sriramula, S., \& Lazartigues, E. (2011). ACE2/ANG-(1-7)/Mas pathway in the brain: the axis of good. Am.J.Physiol Regul.Integr.Comp Physiol, 300, R804-R817.

Xu, S., Zhi, H., Hou, X., Cohen, R. A., \& Jiang, B. (2011). IkappaBbeta attenuates angiotensin II-induced cardiovascular inflammation and fibrosis in mice. Hypertension, 58, 310-316.

Yamamoto, K., Ohishi, M., Katsuya, T., Ito, N., Ikushima, M., Kaibe, M. et al. (2006). Deletion of angiotensin-converting enzyme 2 accelerates pressure overload- 
induced cardiac dysfunction by increasing local angiotensin II. Hypertension, 47, 718726.

Youn, H. J., Rokosh, G., Lester, S. J., Simpson, P., Schiller, N. B., \& Foster, E. (1999). Two-dimensional echocardiography with a 15-MHz transducer is a promising alternative for in vivo measurement of left ventricular mass in mice.

J.Am.Soc.Echocardiogr., 12, 70-75.

Zhong, J., Basu, R., Guo, D., Chow, F. L., Byrns, S., Schuster, M. et al. (2010). Angiotensin-converting enzyme 2 suppresses pathological hypertrophy, myocardial fibrosis, and cardiac dysfunction. Circulation, 122, 717-28, 18.

Zisman, L. S., Keller, R. S., Weaver, B., Lin, Q., Speth, R., Bristow, M. R. et al. (2003). Increased angiotensin-(1-7)-forming activity in failing human heart ventricles: evidence for upregulation of the angiotensin-converting enzyme Homologue ACE2. Circulation, 108, 1707-1712. 